\title{
QTL mapping of melon fruit quality traits using a high-density GBS-based genetic map
}

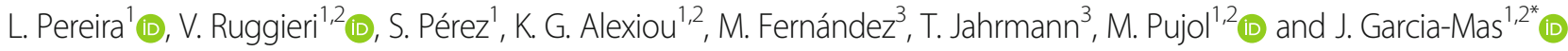

\begin{abstract}
Background: Melon shows a broad diversity in fruit morphology and quality, which is still underexploited in breeding programs. The knowledge of the genetic basis of fruit quality traits is important for identifying new alleles that may be introduced in elite material by highly efficient molecular breeding tools.

Results: In order to identify QTLS controlling fruit quality, a recombinant inbred line population was developed using two commercial cultivars as parental lines: "Védrantais", from the cantalupensis group, and "Piel de Sapo", from the inodorus group. Both have desirable quality traits for the market, but their fruits differ in traits such as rind and flesh color, sugar content, ripening behavior, size and shape. We used a genotyping-by-sequencing strategy to construct a dense genetic map, which included around five thousand variants distributed in 824 bins. The RIL population was phenotyped for quality and morphology traits, and we mapped 33 stable QTLs involved in sugar and carotenoid content, fruit and seed morphology and major loci controlling external color of immature fruit and mottled rind. The median confidence interval of the QTLs was $942 \mathrm{~kb}$, suggesting that the high density of the genetic map helped in increasing the mapping resolution. Some of these intervals contained less than a hundred annotated genes, and an integrative strategy combining gene expression and resequencing data enabled identification of candidate genes for some of these traits.
\end{abstract}

Conclusion: Several QTLs controlling fruit quality traits in melon were identified and delimited to narrow genomic intervals, using a RIL population and a GBS-based genetic map.

Keywords: Quantitative trait loci, Melon, Fruit quality, Fruit morphology, Genotyping-by-sequencing, Genetic map

\section{Background}

Melon (Cucumis melo L.) is an important crop worldwide, with a production of more than 31 million tons in 2016 [1]. The main producers are in temperate regions, with China accounting for around $50 \%$ of total production. Until the last decade, Africa was considered the origin for melon, but recent phylogenetic studies suggest that the species originated in Asia [2]. Traditionally, two subspecies have been described: C. melo ssp. melo, which includes most of the commercial varieties in European markets belonging to cantalupensis and inodorus botanical groups, and C. melo

\footnotetext{
* Correspondence: jordi.garcia@irta.cat

${ }^{1}$ Centre for Research in Agricultural Genomics (CRAG) CSIC-IRTA-UAB-UB, Campus UAB, 08193 Cerdanyola, Barcelona, Spain

${ }^{2}$ IRTA (Institut de Recerca i Tecnologia Agroalimentàries), Campus UAB, 08193 Cerdanyola, Barcelona, Spain

Full list of author information is available at the end of the article
}

ssp. agrestis, which contains most of the Asian exotic landraces and accessions [3]. There is high phenotypic and genetic variability between and within melon subspecies for diverse traits, including plant architecture, sex determination, yield and fruit characteristics [3]. Several mapping populations have been used to study this diversity, as $F_{2}[4$, $5]$, introgression lines (IL) $[6,7]$ and recombinant inbred lines (RIL) [8-10]. Generally, the crosses used to develop these mapping populations have been obtained between exotic (chinensis, conomon, makuwa or flexuosus groups) and cultivated (cantalupensis, reticulatus or inodorus groups) melon types. However, it is of great interest to study the variability between two occidental commercial varieties from different botanical groups, since this has not yet been thoroughly exploited through linkage mapping studies. Association studies using accession panels is another approach that has recently been shown to have the 
potential for characterizing important agronomic traits in melon $[11,12]$.

In addition to the above-mentioned genetic tools, diverse genomic resources have been developed in melon during the past years. Melon is a diploid species with a small genome $(450 \mathrm{Mb})$ and 12 chromosomes $(2 \mathrm{n}=24)$. The use of genomic resources to better understand fruit morphology and quality has been facilitated by the availability of a reference genome [13] and the rapid advances in Next-Generation Sequencing (NGS) technologies, such as RNA-seq [9] and Genotyping-By-Sequencing (GBS) $[11,14,15]$. The GBS strategy is based on the reduction of genome complexity before sequencing, generally through restriction enzyme digestion; only a low percentage of the genome is sequenced but the fragments are normally well distributed across the genome [16]. The GBS approach has been widely used in many species [17-20] due to its simplicity, effectiveness and low-cost when compared to other high-throughput genotyping techniques. The availability of high numbers of SNPs has increased the precision of Quantitative Trait Loci (QTL) mapping. Linkage maps have shown their effectiveness as a tool to study the genetic architecture of both monogenic and complex traits [21]. Recently, high-density maps using hundreds [22] to thousands of markers $[10,14,15]$ have been constructed for QTL mapping of fruit traits. It has been demonstrated that a higher SNP density substantially increases the QTL mapping potential, affecting both the detection and the resolution of QTLs [10, 15].

One of the most important aspects for the market is fruit quality. Fruits from the cantalupensis group are usually defined by medium fruit weight, round shape, climacteric ripening, orange flesh and white, ribbed and netted rind. In contrast, inodorus melons are characterized by non-climacteric ripening, high sugar content, being generally large and elliptical, with green, mottled and smooth rind without ribs nor vein tracts [23]. Several bi-parental mapping and association analyses have been performed for most of these traits, which have been integrated and reviewed by [21]. Some of these traits seem to be under monogenic or oligogenic control [24], such as flesh and rind color, sutures and ribs. However, the most relevant traits related to fruit quality, such as sugar content, fruit size and shape and climacteric ripening are complex and polygenic [10, 25-27]. Extensive research has been done to dissect the genetic control of these traits, but they have been generally limited to crosses between exotic and cultivated material types. Even though they are very valuable, the introduction of exotic alleles in breeding programs is complicated due to linkage drag, with a high cost to remove undesired regions [28]. However, the variation between phylogenetically close but phenotypically different commercial cultivars has not been exploited previously, and can offer new tools easily implemented in breeding programs. The aim of this study was to identify QTLs and major loci related with fruit quality in narrow genomic intervals, using a high-density genetic map obtained with a RIL population from a cantalupensis $\mathrm{x}$ inodorus cross.

\section{Results}

\section{Phenotyping of the RIL population}

The RIL population and the parental lines were evaluated during the summers of 2015 (blocks T1-T3) and 2016 (blocks T4-T5). Several interesting traits related with fruit quality and morphology segregated in the population. Some of these were considered as qualitative (Table 1), although some variation in intensity was observed for MOT, ECOL and YELL. These traits were evaluated for their segregation ratio in the RIL population (Table 1). A segregation of 1:1, expected for a monogenic trait, was observed for ECOL, where the white color of Ved was dominant over green. For MOT, the segregation showed a deviation from the 1:1 expected for a monogenic trait, and the mottled pattern of PS was dominant over its absence. For YELL, a segregation of 3:1 (yellow: not yellow) was observed, in accordance with a dominant epistasis system, where presence of the yellowing allele was dominant.

The phenotypic values for the quantitative traits are shown in Table 2. In each block, we included the parental lines (Ved, PS) and the Hyb as controls. As an example, fruit weight was lower and quite stable in Ved (771 $\pm 156 \mathrm{~g})$ when compared to PS and Hyb (1311 \pm 428

Table 1 Mapping of qualitative traits in the "Védrantais" x "Piel de Sapo" Recombinant Inbred Line population

\begin{tabular}{|c|c|c|c|c|c|c|c|c|}
\hline Trait & PS & Ved & Hyb & Expected segregation & $x^{2}$ & Map position (CM) & $\mid$ Interval| & Gene (reference) \\
\hline $\begin{array}{l}\text { External color of } \\
\text { immature fruit (ECOL) }\end{array}$ & Green & White & White & $1: 1$ & $1.39 \mathrm{~ns}$ & 39.8 & $\begin{array}{l}\text { chr07_2707033- } \\
\text { chr07_4345823 }\end{array}$ & Wi [44] \\
\hline Mottled rind (MOT) & Yes & No & Yes & $1: 1$ & $6.22^{*}$ & 127.7 & $\begin{array}{l}\text { chr02_26206397- } \\
\text { end of chr02 }\end{array}$ & $M t-2[45]$ \\
\hline \multirow[t]{2}{*}{$\begin{array}{l}\text { Yellowing of mature } \\
\text { rind (YELL) }\end{array}$} & Yes & No & Yes & $3: 1$ & $1.38 \mathrm{~ns}$ & 34.1 & $\begin{array}{l}\text { chr10_3152004- } \\
\text { chr10_4144573 }\end{array}$ & CmKFB [46] \\
\hline & & & & & & 125.1 & $\begin{array}{l}\text { chr05_28951742- } \\
\text { chr05_29246933 }\end{array}$ & This work \\
\hline
\end{tabular}

${ }^{a}$ According to version v3.6.1 of melon reference genome 
Table 2 Mean and standard deviation of the parental lines and mean and range in the Recombinant Inbred Line population for each quantitative trait

\begin{tabular}{|c|c|c|c|c|c|c|}
\hline \multirow[t]{2}{*}{ Class } & \multirow[t]{2}{*}{ Trait (unit) } & \multicolumn{3}{|c|}{ Parental lines } & \multicolumn{2}{|c|}{ RIL population } \\
\hline & & PS & Ved & Hyb & Mean & Range \\
\hline Fruit quality & SSC ( ${ }^{\circ}$ Brix) & $11.8 \pm 1.3$ & $10.7 \pm 0.8$ & $10.6 \pm 1.7$ & 10.4 & $5.6-14.0$ \\
\hline \multirow[t]{5}{*}{ Fruit morphology } & Weight (FW) (g) & $1311 \pm 428$ & $771 \pm 156$ & $1148 \pm 387$ & 994 & $345-1763$ \\
\hline & Diameter (FD) (cm) & $13.0 \pm 1.3$ & $11.7 \pm 0.6$ & $12.8 \pm 1.3$ & 12.0 & $8.3-14.8$ \\
\hline & Shape (FS) & $1.36 \pm 0.21$ & $1.02 \pm 0.22$ & $1.07 \pm 0.06$ & 1.2 & $0.9-1.6$ \\
\hline & Length $(\mathrm{FL})(\mathrm{cm})$ & $17.8 \pm 3.5$ & $12.0 \pm 2.8$ & $13.8 \pm 2.1$ & 14.0 & $9.5-19.3$ \\
\hline & Perimeter (FP) (cm) & $51.5 \pm 7.3$ & $39.8 \pm 4.9$ & $45.1 \pm 5.9$ & 44.0 & $30.7-53.6$ \\
\hline Flesh color & Carotenoid content $(\mathrm{CAR})(\mu \mathrm{g} / \mathrm{gFW})^{\mathrm{a}}$ & $0.7 \pm 0.2$ & $18.4 \pm 5.6$ & $10.9 \pm 1.4$ & 8.8 & $0.4-30.6$ \\
\hline \multirow[t]{2}{*}{ Seed morphology } & Seed weight (SW) (mg) & $31 \pm 4$ & $30 \pm 3$ & $37 \pm 9$ & 32 & $18-45$ \\
\hline & Seed number (SN) & $249 \pm 114$ & $324 \pm 108$ & $408 \pm 194$ & 293 & $67-499$ \\
\hline
\end{tabular}

${ }^{\mathrm{a} O n l y}$ blocks $\mathrm{T} 1, \mathrm{~T} 2$ and $\mathrm{T} 3$ were analyzed

and $1148 \pm 387 \mathrm{~g}$, respectively), with some individuals doubling the weight but showing a higher dispersion (Fig. 1b). The dispersion can be observed in the standard deviation, which is high in complex traits with low heritability (e.g. SSC, FW) and low in more stable traits (e.g. FS) (Table 2, Additional file 1: Figure S1). Transgressive segregation was observed for all traits analyzed.

The distribution of the data for each trait and block was represented with beanplots (Fig. 1c, Additional file 2: Figure S2). The distribution was normal in all blocks for SSC, FL and FS but for FW, FD, FP, SW and SN the deviation from normal was significant in at least one block. CAR was not normally distributed in any of the three blocks analyzed, with more individuals having high-carotenoid content values $(>5 \mu \mathrm{g} / \mathrm{g}$ FW) than intermediate values.

The correlations between traits are presented in Fig. 2. There was a clear relationship within morphometric measurements. As expected, correlation between fruit dimensions (FL, FD and FP) and FW was strong and positive. The correlation between FS and FL was higher than with FD, implying that length is the major determinant of fruit shape in this population. A positive correlation was detected between seed $(\mathrm{SN}, \mathrm{SW})$ and some fruit morphometric traits (FP, FW, FD). ECOL correlated negatively with CAR and positively with FL, FP, FW and FD. YELL negatively correlated with SSC.

\section{Construction of a genetic linkage map through genotyping-by-sequencing}

Sequencing of 91 GBS libraries for the 89 RILs and the two parental lines yielded about 230 million raw reads, corresponding to an average of 2.5 million reads/sample. About $86 \%$ of the reads were successfully mapped onto the melon genome (version 3.6.1). A total of 125,465 raw GBS-polymorphisms were called with Fast-GBS. However, about $80 \%$ of them were removed due to lack of agreement when compared to the variants from the published re-sequencing data of Ved and PS [36]. The remaining 24,988 pre-filtered variants were further reduced by applying additional filtering criteria (see Material and Methods). In particular, about 50\% of the variants were filtered out due to a more restrictive missing value threshold imposed (MV $\leq 60 \%$ ) and about $20 \%$ due to the other criteria imposed (at least one homozygous variant for marker, global quality >100, only bi-allelic variants). Among the 5944 variants retained, 9.8\% were INDEL and 90.2\% were SNPs, supported by an average coverage of 17.89. An average of 492 variants per chromosome was detected and chromosome six harbored the highest number (Table 3). A high correlation was observed between the number of variants per chromosome and their physical size. This highlighted that the variants were well distributed and quite uniformly covered all the chromosomes. The distribution of the markers along the 12 melon chromosomes and the unassembled scaffolds (chromosome 0 ) is given in Table S1a (Additional file 3). A further manual refinement of the marker dataset was carried out to ensure high reliability for the genetic map construction and the QTL mapping analysis, discarding 1056 markers (Additional file 3: Table S1b and Table 3). The 4888 retained GBS-markers were used to build individual bins (Additional file 3: Table S1c). Excluding the bins differing only because of the presence of heterozygous variants, we obtained a total of 824 GBS-derived bins to build the genetic linkage map.

The genetic distance, the covered physical distance and the recombination rate of the genetic map are presented in Table 3. The map covered $1519.4 \mathrm{cM}$, distributed in 13 linkage groups (LG) (Fig. 3). Two of them belong to chromosome XI, which was split in two linkage groups LG XIa and LG XIb. The largest LG, 156.9 cM, was LG IV and the smallest one, 103.2 cM, LG X. In 

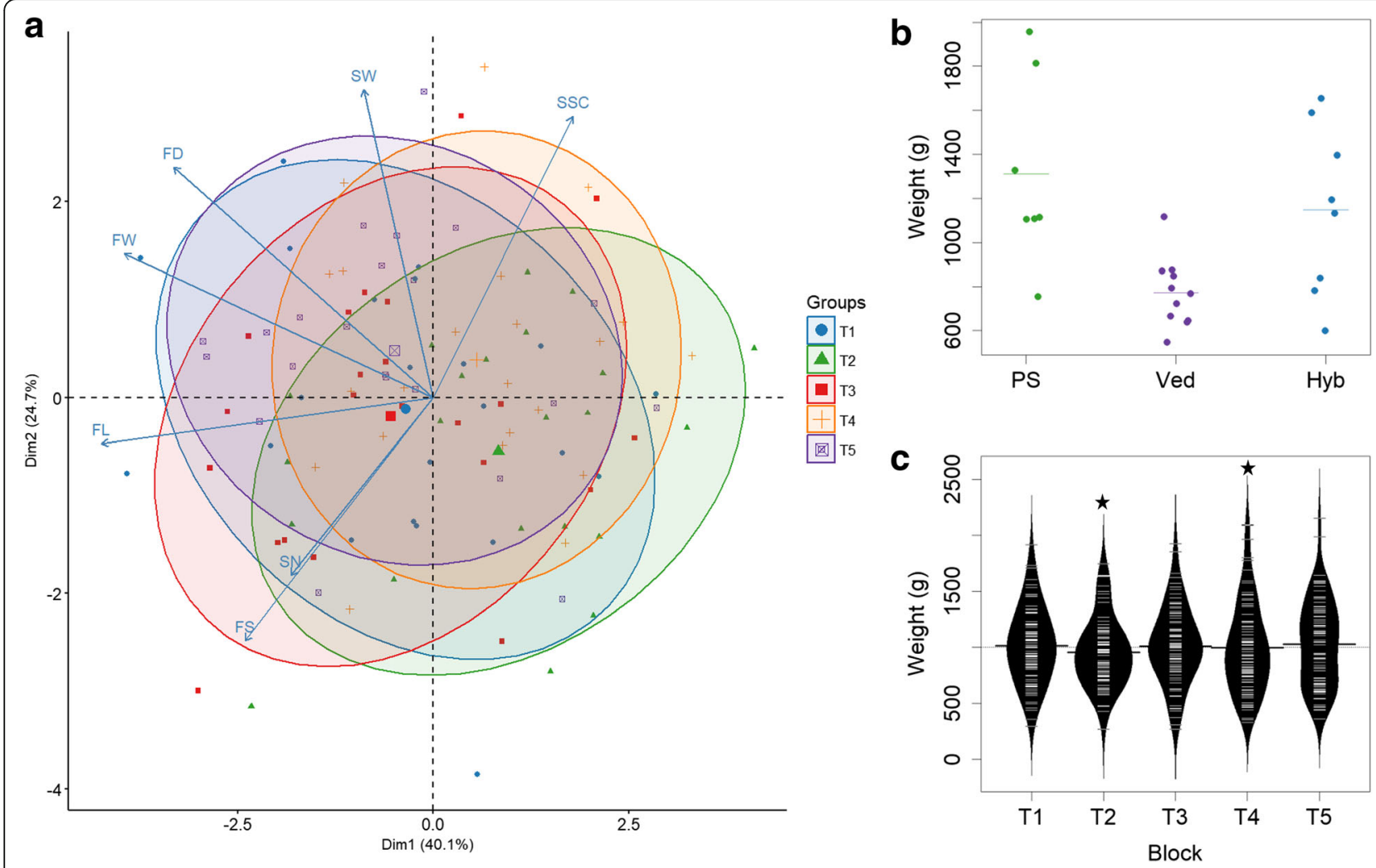

Fig. 1 Phenotypic data in the RIL population and the parental lines. a PCA showing the similarities between blocks T1 to T5. b Distribution of fruit weight in the parental lines, merging data from all blocks. Each dot corresponds to an observation in any of the five blocks T1-T5. The mean for each line is shown with a horizontal line. c Distribution of fruit weight in the RIL population in each block T1-T5; black stars indicate significant deviations from normality

terms of physical distance, we calculated the covered region for each chromosome as the difference between the physical positions of the last and the first markers in the LG. The map covered $97 \%$ of the melon genome. LG I had the most coverage, with $98.97 \%$ of the physical sequence covered by markers, and LG X the least, with coverage of only $87.72 \%$ of the sequence represented in the genetic map.

In the genetic map we included nine bins that mapped to chromosome 0 , which may help in anchoring additional scaffolds to pseudomolecules. These bins belong to LG I, LG II, LG III, LG V, LG VIII, LG IX and LG XII. We also detected a few inconsistencies between the physical and the genetic map: three bins from chromosomes 2 and 7 according to their physical position were inserted in LG X (not shown).

\section{Mapping of major loci and QTLs Qualitative traits}

For two of the three qualitative traits studied in our RIL population, MOT and ECOL, we detected one major locus controlling the phenotype in LGs II and VII, respectively. In the case of YELL, two minor QTLs in addition to a major locus were observed. In Fig. 4, we show the phenotypical differences between the two categorical classes for each trait and the association between the markers and the phenotype using the non-parametric KW test. In all cases, interval mapping was used to confirm that the results were consistent using both methodologies.

According to the segregation data, ECOL showed monogenic inheritance. This hypothesis was confirmed with the mapping experiments. The gene conferring the external color of immature fruit was located in LG VII, with a KW value of 81.02 at position chr07_4193950 (Fig. 4a). In the interval mapping, a major QTL with maximum LOD of 72.80 at $39.8 \mathrm{cM}$ in LG VII, corresponding to the same physical position as KW, explained $97.7 \%$ of the variance and was delimited in a region of $1.6 \mathrm{Mb}$ (Table 1). We detected a second QTL ECOLQU3.1 with a significant LOD score at $113.4 \mathrm{cM}$ in LG III (Table 4), with an additive effect of 0.2 (greener skin when the Ved allele was present); this QTL can also be seen in Fig. 4a, although with a lower KW value.

The evaluation of MOT was difficult in some fruits. The allele that confers the mottled rind is from PS, but it is not easily detected due to the dark green color of the PS rind, which masks the darker spots 


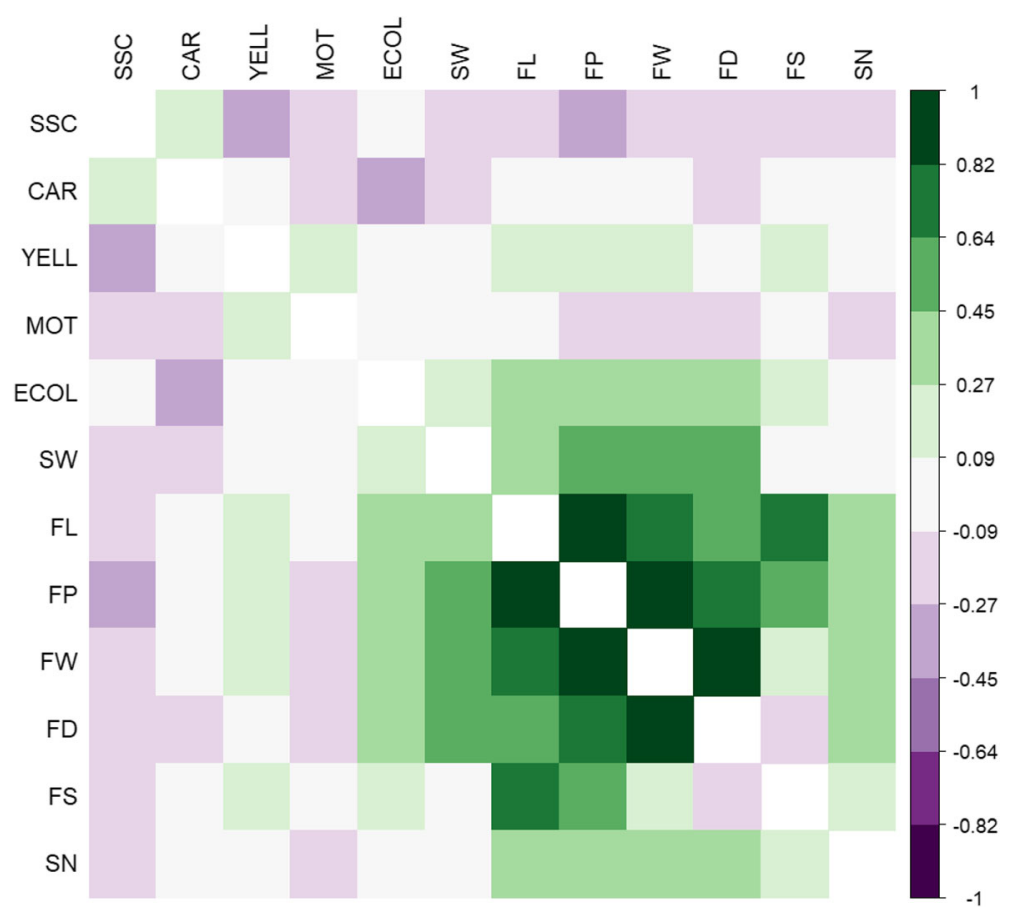

Fig. 2 Correlation matrix between the measured traits. The scale represent the values of Pearson coefficient between the traits using the mean value across the blocks for each variable

(Additional file 4: Figure S3a). In contrast to the striking appearance of dark spots in melons with white rind (Fig. 4b). Although the segregation did not follow the expected 1:1 ratio for a monogenic trait (Table 1), the $\mathrm{KW}$ test and interval mapping clearly showed a major locus in the distal part of LG II (Fig. 4b). In fact, after analyzing the segregation of the markers in this region of the genetic map, we observed a segregation distortion $\left(x^{2}=6.40\right.$ in the closest marker). The major locus is at the end of the LG, with chr02_26206397 the last

Table 3 Variants from the SNP calling and characteristics of the genetic map by chromosome

\begin{tabular}{|c|c|c|c|c|c|c|c|c|c|}
\hline \multirow[t]{2}{*}{$\overline{C h r^{a}}$} & \multicolumn{5}{|c|}{ Number of variants } & \multirow{2}{*}{$\begin{array}{l}\text { Genetic } \\
\text { distance (cM) }\end{array}$} & \multirow{2}{*}{$\begin{array}{l}\text { Total physical } \\
\text { distance }(\mathrm{bp})^{c}\end{array}$} & \multirow{2}{*}{$\begin{array}{l}\text { Covered physical } \\
\text { distance }(b p)^{d}\end{array}$} & \multirow{2}{*}{$\begin{array}{l}\text { Recombination } \\
\text { rate (cM/Mb) }\end{array}$} \\
\hline & Raw data & Pre-filtered & Filtered & Genetic map & $\mathrm{N}^{\circ}$ bins & & & & \\
\hline 1 & 6584 & 2087 & 417 & 360 & 63 & 124.6 & $37,037,532$ & $36,657,204$ & 3.40 \\
\hline 2 & 9643 & 2511 & 616 & 510 & 71 & 127.7 & $27,064,691$ & $26,042,194$ & 4.90 \\
\hline 3 & 12,136 & 2663 & 622 & 508 & 81 & 122.6 & $31,666,927$ & $31,095,866$ & 3.94 \\
\hline 4 & 15,054 & 2122 & 534 & 440 & 97 & 156.9 & $34,318,044$ & $33,448,353$ & 4.69 \\
\hline 5 & 9007 & 1754 & 466 & 365 & 70 & 125.1 & $29,324,171$ & $28,833,706$ & 4.34 \\
\hline 6 & 9067 & 2575 & 638 & 501 & 79 & 152.9 & $38,297,372$ & $37,423,280$ & 4.09 \\
\hline 7 & 13,792 & 2062 & 539 & 460 & 78 & 130.9 & $28,958,359$ & $28,560,617$ & 4.58 \\
\hline 8 & 8242 & 2281 & 519 & 400 & 67 & 129.2 & $34,765,488$ & $32,947,662$ & 3.92 \\
\hline 9 & 6157 & 1716 & 419 & 369 & 54 & 109.5 & $25,243,276$ & $24,844,222$ & 4.41 \\
\hline 10 & 10,363 & 1776 & 383 & 319 & 45 & 103.2 & $26,663,822$ & $23,388,534$ & 4.41 \\
\hline 11 & 14,194 & 1801 & 380 & 326 & 52 & $24+109^{b}$ & $34,457,057$ & $33,905,267$ & 3.92 \\
\hline 12 & 11,226 & 1640 & 360 & 310 & 58 & 103.8 & $27,563,660$ & $26,974,440$ & 3.85 \\
\hline 0 & - & - & 51 & 20 & 9 & - & - & - & - \\
\hline Total & 125,465 & 24,988 & 5944 & 4888 & 824 & 1519.4 & $375,360,399$ & $367,540,170$ & - \\
\hline
\end{tabular}

\section{${ }^{\mathrm{a}}$ Melon chromosome}

${ }^{\mathrm{b}}$ Chromosome $\mathrm{XI}$ is divided in two linkage groups

'Version 3.6.1 of the melon genome

${ }^{\mathrm{d}}$ Subtraction between the first and the last positions covered by markers in the genetic map 


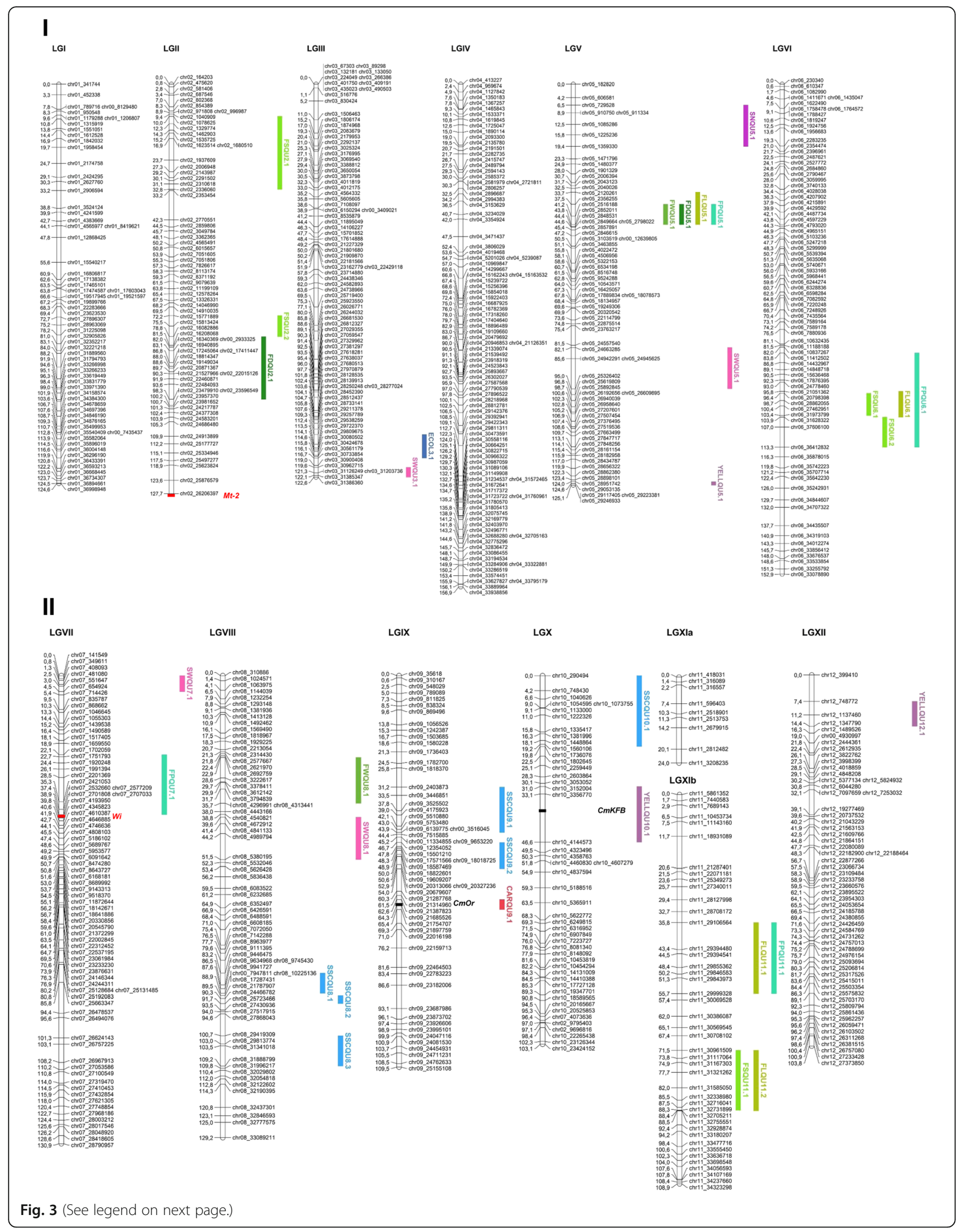


(See figure on previous page.)

Fig. 3 Genetic map containing detected QTLs and major loci. Major loci Mt-2 (MOT) and Wi (ECOL) are placed in their physical position, indicated with a red line. Cloned genes CmOr and CMKFB are placed in their physical position, indicated with a black line. QTLs are represented as colored bars, using a 1-LOD confidence interval. Green tones for morphological QTLS (FP, FW, FS, FL and FD), pink tones for seed traits (SN and SW), dark blue for ECOL, light blue for SSC, red for CAR and purple for YELL

marker of the linkage group and the most associated to the phenotype, with a LOD $=63.90$ in interval mapping. A region in the melon genome of approximately $0.8 \mathrm{Mb}$ distal to chr02_26206397, not covered by markers in the genetic map, was considered in the QTL interval (Table 1).

Another qualitative trait evaluated was the yellowing of mature rind (YELL). As with the mottled rind, the yellow allele comes from PS. It is partially masked by the dark green rind color, but has a different tonality, leading to a greyish color which is visible when the yellow allele is absent (Additional file 4: Figure S3b). The observed segregation suggested the hypothesis of two genes under dominant epistasis. The first and most important gene is in LG X, in a region of approximately 1 Mb (Table 1 and Table 4) and was detected in the KW test (Fig. 4c) and in interval mapping with a LOD $=8.79$. Two other QTLs were detected: YELLQU5.1 in LG V, explaining $15.1 \%$ of the variance with the Ved allele decreasing the yellow color; and YELLQU12.1 in LG XII, explaining $14.2 \%$ of the variance with the Ved allele increasing the yellow color. Both QTLs can also be observed in Fig. 4a, although with lower KW values.

\section{Quantitative traits}

QTL mapping was performed using the mean of five blocks and using each block individually (T1-T5) (Table 4, Fig. 3). A QTL was considered significant with a LOD score higher than 2.5 in the mapping analysis that considered the mean phenotypic values. We also show the LOD for the same QTL/position in the individual blocks. Thirty-three significant QTLs were detected for the 11 measured traits. The level of consistency between blocks depended on the trait and the significance of the QTL. Although some QTLs seemed to be dependent on the year, for example YELLQU12.1, with higher LOD scores in both 2016 blocks than in the three 2015 blocks, although this effect was not general.

Fruit quality traits We evaluated SSC, an important trait concerning fruit quality in melon. Both parental lines are commercial types and the SSC is acceptable,
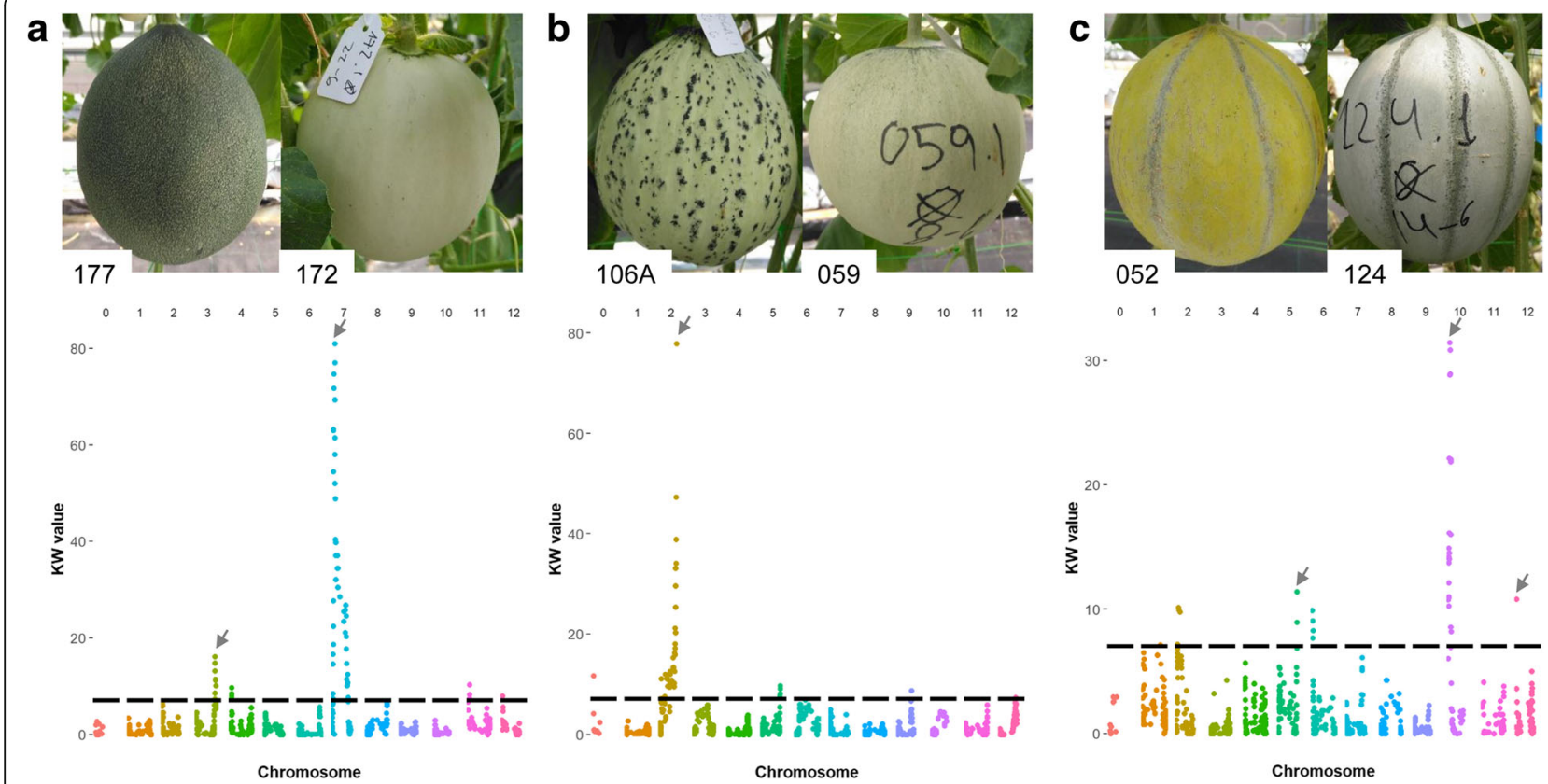

Fig. 4 Kruskal-Wallis (KW) statistics test (significant threshold for $p<0,01$ ) and photos of fruits showing the two observed phenotypes for ECOL, MOT and YELL. Grey arrows indicate the most significant values. Chromosomes ( 0 to 12 from left to right) are represented with different colors. a External color of immature fruit (ECOL) (RIL 177 green and RIL 172 white). b Mottled rind (MOT) (RIL 106A presence and RIL 059 absence). c Yellowing of mature rind (YELL) (RIL 052 presence and RIL 124 absence) 
Table 4 QTL analysis for the traits evaluated. QTLs with LOD > 2.5 using the mean of five blocks (maximum LOD in each block T1-T5 is also annotated)

\begin{tabular}{|c|c|c|c|c|c|c|c|c|c|c|c|c|c|c|}
\hline Trait & QTL ID & LOD & $R^{2}$ & $\begin{array}{l}\text { Additive } \\
\text { effect }^{\mathrm{a}}\end{array}$ & Chr & $\begin{array}{l}\text { Genetic } \\
\text { position (cM) }\end{array}$ & $\begin{array}{l}\text { Physical } \\
\text { position }^{\mathrm{b}}(\mathrm{pb})\end{array}$ & $\begin{array}{l}\text { Flanking marker } 1 \\
\text { respect 1-LOD Cl }\end{array}$ & $\begin{array}{l}\text { Flanking marker } 2 \\
\text { respect to 1-LOD Cl }\end{array}$ & $\begin{array}{l}\mathrm{LOD} \\
\mathrm{T} 1^{\mathrm{C}}\end{array}$ & $\begin{array}{l}\mathrm{LOD} \\
\mathrm{T} 2^{\mathrm{C}}\end{array}$ & $\begin{array}{l}\text { LOD } \\
\text { T3 }^{C}\end{array}$ & $\begin{array}{l}\mathrm{LOD} \\
\mathrm{T}^{\mathrm{C}}\end{array}$ & $\begin{array}{l}\text { LOD } \\
T^{c}\end{array}$ \\
\hline \multirow[t]{6}{*}{ SSC } & SSCQU8.1 & 9.96 & 40.3 & -1.26 & 8 & 86.49 & $9,634,968$ & chr08_9446475 & chr08_17287431 & 6.3 & 8.0 & 2.7 & 5.8 & 44 \\
\hline & CQU8.2 & 9.83 & 39.9 & 1.27 & 8 & 90.28 & $2,446,682$ & 87907 & chr08 & .7 & 8.9 & $\underline{3.0}$ & 5.9 & 4.7 \\
\hline & SSCQU8.3 & 10.76 & 42.7 & -1.3 & 8 & 102.66 & $29,813,774$ & chr08_29419309 & chr08_31888799 & 8.2 & 6.2 & 3.8 & 6.9 & 5. \\
\hline & SSCQU9.1 & 2.78 & 13.4 & 0.711 & 9 & 33.49 & $3,446,851$ & chr09_2403873 & chr09_6139775 & 1.6 & $\underline{4.1}$ & 1.4 & $\underline{2.7}$ & 25 \\
\hline & CQU9.2 & 2.57 & 12.5 & 0.69 & 9 & 49.89 & $18,822,601$ & chr09_12354052 & chr09_20679607 & 0.8 & $\underline{4.6}$ & 1.5 & .5 & 2.7 \\
\hline & SSCQU10.1 & 3.3 & 15.7 & 0.77 & 10 & 18.06 & $1,448,864$ & chr10_290494 & chr10_1736076 & 1.5 & 2.8 & 1.5 & 2.6 & 2.6 \\
\hline \multirow[t]{2}{*}{ FW } & FWQU5.1 & 6.42 & 28.3 & 153.27 & 5 & 40.51 & $2,516,188$ & chr05_2356255 & chr05_2852011 & 6.7 & 2.1 & 2.3 & $\underline{3.0}$ & 2 \\
\hline & FWQU8.1 & 2.51 & 12.2 & -92.38 & 8 & 31.73 & $3,794,839$ & chr08_2692759 & chr08_4296991 & 0.8 & 0.3 & 1.7 & $\underline{2.5}$ & $\underline{2.8}$ \\
\hline \multirow[t]{2}{*}{ FD } & FDQU2.1 & 3.29 & 15.6 & 0.47 & 2 & 88.58 & $19,149,034$ & chr02_16082886 & chr02_23479910 & 1.4 & 1.4 & 1.7 & $\underline{3.2}$ & 2.4 \\
\hline & FDQU5.1 & 4.92 & 22.5 & 0.6 & 5 & 41.22 & $2,516,188$ & chr05_2356255 & chr05_2852011 & $\underline{5.5}$ & 1.8 & 2.3 & 2.0 & 1.5 \\
\hline \multirow[t]{5}{*}{ FS } & FSQU2.1 & 2.96 & 14.2 & -0.061 & 2 & 30.07 & $2,291,502$ & chr02_1078625 & chr02_2336060 & $\underline{2.6}$ & $\underline{3.0}$ & 2.4 & $\underline{3.6}$ & 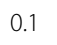 \\
\hline & FSQU2.2 & 3.18 & 15.2 & -0.064 & 2 & 75.52 & $15,813,424$ & chr02_15771889 & chr02_16082886 & $\underline{4.5}$ & 2.0 & 0.3 & 1.5 & 1.4 \\
\hline & FSQU6.1 & 7.7 & 32.9 & -0.092 & 6 & 99.72 & $27,462,954$ & chr06_20798398 & chr06_31973799 & $\underline{4.1}$ & $\underline{4.9}$ & $\underline{6.9}$ & $\underline{2.5}$ & $\underline{5.7}$ \\
\hline & FSQU6.2 & 6.96 & 30.2 & -0.087 & 6 & 106.99 & $37,606,100$ & chr06_31628322 & chr06_36412832 & $\underline{4.3}$ & $\underline{5.0}$ & $\underline{5.6}$ & $\underline{2.9}$ & $\underline{3.4}$ \\
\hline & FSQU11.1 & 3.34 & 15.9 & -0.064 & $11 b$ & 81.68 & $31,585,050$ & chr11_30961509 & chr11_32731899 & 2.1 & 2.8 & $\underline{4.8}$ & 2.1 & 1.1 \\
\hline \multirow[t]{4}{*}{$\mathrm{FL}$} & FLQU5.1 & 5.29 & 24 & 1.16 & 5 & 40.51 & 88 & 20261 & chrc & $\underline{5.1}$ & 2.3 & 2.0 & $\underline{3.8}$ & $\underline{2.9}$ \\
\hline & FLQU6.1 & 5.78 & 25.9 & -1.1 & 6 & 100.43 & $27,462,951$ & chr06_21051362 & chr06_31628322 & $\underline{3.2}$ & $\underline{4.5}$ & $\underline{3.8}$ & $\underline{2.5}$ & $\underline{4.6}$ \\
\hline & FLQU11.1 & 3.79 & 17.8 & -0.96 & $11 \mathrm{~b}$ & 47.46 & $29,855,362$ & chr11_29106564 & chr11_29999328 & $\underline{3.7}$ & $\underline{2.5}$ & 1.7 & $\underline{3.1}$ & 1.8 \\
\hline & FLQU11.2 & 2.67 & 12.9 & -0.81 & $11 \mathrm{~b}$ & 80.68 & $31,585,050$ & chr11_30961509 & chr11_32755551 & $\underline{4.7}$ & $\underline{2.8}$ & $\underline{4.4}$ & 0.6 & 0.2 \\
\hline \multirow[t]{4}{*}{ FP } & FPQU5.1 & 6.73 & 29.4 & 2.95 & 5 & 40.51 & $2,516,188$ & chr05_2356255 & chr05_2852011 & $\underline{7.7}$ & 2.8 & 2.6 & $\underline{3.2}$ & $\underline{2.5}$ \\
\hline & FPQU6.1 & 2.75 & 13.3 & -1.81 & 6 & 100.43 & $27,462,951$ & chr06_11412502 & chr06_36412832 & 1.5 & $\underline{3.2}$ & 1.6 & 1.7 & 2.0 \\
\hline & FPQU7.1 & 2.54 & 12.3 & -1.83 & 7 & 29.47 & $2,201,369$ & chr07_1702059 & chr07_2701808 & 1.0 & 1.8 & 1.1 & 2.4 & 1.7 \\
\hline & FPQU11.1 & 3.27 & 15.6 & -2.03 & $11 \mathrm{~b}$ & 42.79 & $9,394,480$ & chr11_29106564 & chr11_29999328 & $\underline{3.5}$ & 2.7 & 1.1 & $\underline{3.0}$ & 1.1 \\
\hline \multirow[t]{3}{*}{ YELL } & YELLQU5.1 & 3.15 & 15.1 & -0.15 & 5 & 125.12 & $29,117,405$ & chr05_28951742 & chr05_29246933 & $\underline{3.8}$ & 2.8 & 1.4 & $\underline{2.5}$ & 1.9 \\
\hline & YELLQU10.1 & 8.79 & 36.6 & -0.25 & 10 & 34.07 & $3,356,770$ & chr10_3152004 & chr10_4144573 & $\underline{8.1}$ & $\underline{5.9}$ & $\underline{4.2}$ & $\underline{6.6}$ & $\underline{6.7}$ \\
\hline & YELLQU12.1 & 2.96 & 14.2 & 0.15 & 12 & 11.21 & $1,137,460$ & chr12_748772 & chr12_1347790 & 1.7 & 1.6 & 1.4 & $\underline{2.8}$ & 2.4 \\
\hline $\mathrm{COL}$ & ECOLQU3.1 & 3.49 & 16.5 & 0.21 & 3 & 113.40 & $29,722,370$ & chr03_29257789 & chr03_30733854 & & $3.5^{\mathrm{d}}$ & & $3.0^{d}$ & \\
\hline CAR & CARQU9.1 & 16.82 & 58.5 & 5.6 & 9 & 64.32 & $21,685,526$ & chr09_21387823 & chr09_21754707 & 12.7 & 11.6 & 13.6 & - & - \\
\hline SN & SNQU5.1 & 2.73 & 13.2 & 33.43 & 5 & 14.46 & $1,225,236$ & chr05_729528 & chr05_1359330 & 2.1 & 0.5 & 0.3 & 2.1 & 0.4 \\
\hline \multirow[t]{4}{*}{ SW } & SWQU3.1 & 4.11 & 19.2 & 2.7 & 3 & 122.11 & $31,385,347$ & chr03_30962715 & chr03_31386360 & 2.4 & 1.6 & 0.3 & $\underline{2.7}$ & 1.7 \\
\hline & SWQU5.1 & 4.3 & 19.9 & 2.9 & 5 & 87.63 & $24,945,625$ & chr05_24663285 & chr05_25326402 & $\underline{3.0}$ & 2.6 & $\underline{3.2}$ & 2.4 & 1.2 \\
\hline & SWQU7.1 & 2.91 & 14.0 & -2.2 & 7 & 0.78 & 349,611 & chr07_141549 & chr07_654924 & 0.9 & 1.4 & 1.5 & 0.9 & 2.0 \\
\hline & SWQU8.1 & 4.29 & 19.9 & -2.7 & 8 & 43.35 & $4,989,794$ & chr08_4672912 & chr08_5308195 & 0.9 & 6.9 & 1.9 & 3.1 & 1.2 \\
\hline
\end{tabular}

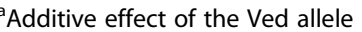

bPhysical position is relative to the melon genome sequence v3.6.1

'Bold and underlined font for LOD scores above 3, underlined font for LOD scores between 2.5-3

dLOD scores for 2015 and 2016, respectively

but in PS it is slightly higher than in Ved. In our evaluations, it ranged from 10.5 to $13.1^{\circ}$ Brix in PS and from 9.9 to $11.5^{\circ}$ Brix in Ved. The hybrid was similar to Ved (Table 2 and Additional file 1: Figure S1).

Six significant QTLs were detected for SSC (Table 4). Among them, SSCQU8.3 was the most consistent, with a LOD score above 3.5 in all experiments; it explained $42.7 \%$ of the variance and the Ved allele presented reduced $1.3^{\circ}$ Brix. The QTL is located in LG VIII around $102.66 \mathrm{cM}$, in an interval of $2.5 \mathrm{Mb}$.

Although SSC content is higher in PS than in Ved, in three out of six QTLs the Ved allele had a positive effect, explaining the transgressive segregation observed in the RIL population. The percentage of variance explained by each of them was around $13 \%$ and the additive effect of the Ved allele was $0.7^{\circ}$ Brix. 
Fruit morphology traits We evaluated five traits related with fruit morphology: weight, diameter, shape, length and perimeter. In total, we detected 17 QTLs; some were exclusive for a single trait (e.g. FSQU2.1) and others co-localized for several morphological traits (e.g. FWQU5.1, FDQU5.1, FLQU5.1 and FPQU5.1) (Fig. 3, Table 4).

The most significant QTL for FW was FWQU5.1 in LG $\mathrm{V}$, explaining $28.3 \%$ of the variance; the allele of Ved increased average fruit weight to $153.27 \mathrm{~g}$. It was detected in the mean analysis with a LOD score of 6.42 and in T1 and $\mathrm{T} 4$ with $\mathrm{LOD} \geq 3$. A QTL in the same interval was also detected with high LOD scores for FD, FL and FP, indicating a major effect on fruit size in this region. The best resolution for this QTL was obtained for FWQU5.1, FDQU5.1 and FPQU5.1, which delimited it to a 500-kb interval.

FLQU6.1 in position $100.4 \mathrm{cM}$ of LG VI, had a LOD score of 5.78 in the mean analysis and was significant in all blocks (T1-T5). It explained $25.9 \%$ of the variance and the Ved allele decreased the length of the fruit by $1.1 \mathrm{~cm}$. The QTL was located in an interval of $10.6 \mathrm{Mb}$ in the centromeric region of the chromosome.

Concerning FS, we describe five QTLs and in all cases, the Ved allele decreased the shape index to produce rounder fruits. A QTL co-localizing with FLQU6.1, FSQU6.1, was the most significant and consistent. FSQU2.1, in a region of approximately $1.3 \mathrm{Mb}$ in LG II, did not co-localize with any other morphological trait-associated QTL.

Flesh color traits Although the gene determining orange flesh color, $\mathrm{CmOr}$, has already been described by [47], we decided to measure total carotenoids content in flesh of ripe fruit. We observed a transgressive segregation (Table 2), finding almost double the total carotenoids as the mean of Ved in some RILs. The QTL mapping revealed just one major QTL in LG IX with a LOD = 16.82 explaining $58 \%$ of the variance (CARQU9.1, Fig. 3). We did not detect any other minor QTL for this trait.

Seed traits Although the mean values for the parental lines were similar, we detected four significant QTLs for seed weight (Table 4, Fig. 3). None of the QTLs for seed weight co-localized with fruit morphology QTLs. The most significant was SWQU8.1, with a LOD score of 4.29, explaining $19.9 \%$ of the variance in the RIL population. The Ved allele diminished seed weight. SWQU8.1 was located in a region of $636 \mathrm{~kb}$. A single QTL SNQU5.1 for seed number was detected in LG V, but with a lower LOD score.

\section{Discussion}

The GBS approach applied in a biparental RIL population is highly effective for QTL mapping studies

Understanding the genetic control of important agronomic traits has been a challenge over the last few decades. Different strategies have shown their effectiveness, but the most used is the QTL mapping approach. Type and size of the population and map density are the main limiting factors for detecting QTLs and their resolution. RIL populations present some advantages: the lines are fixed, so multiple evaluations in different years or environments are possible; each individual has potentially suffered multiple recombination events, increasing the mapping resolution; and the development of this type of population is simple and of low cost using a single-seed descent method without need for intermediate genotyping [48].

Until recently, the main limiting factor, in terms of work and cost, was marker discovery and genotyping. The first genetic maps used during the eighties and nineties generally included from tens to a few hundred markers, mainly isoenzymes and RFLPs [49-51]. Due to the fast development of sequencing technologies and bioinformatics, genotyping is becoming more and more affordable and accessible to the scientific community. A reference genome sequence has already been published for many important crops, including maize [52], rice [53] and tomato [54], among others, facilitating the use of high-throughput genotyping methods based on NGS. The GBS strategy is by far the most widespread technique for high-throughput genotyping, allowing simultaneous variant calling and genotyping for thousands of SNPs and INDELs, and without the need for a reference genome. In melon, GBS has recently been used to characterize collections of accessions $[11,14,55]$ and biparental populations $[14,15]$. The number of variants we obtained (24,988 SNPs and INDELs) was comparable to those obtained in these previous studies, ranging from 13,756 to 99,263 . Such a divergence in the number of variants is expected, depending on factors such as the diverse origin of the germplasm, the sequencing technology used, the software chosen for variant calling and the filtering criteria applied. Also the possibility to impute or not the missing values could greatly affect the final number of variants. The number of bins found in our Ved x PS linkage map (824) was lower than in previous studies, 1837 [14] and 2493 [15] respectively. This discrepancy was expected since in those studies the founding cross for the RIL population was between C. melo spp. melo and C. melo ssp. agrestis accessions, which represent wider diversity in comparison to our population.

Although many QTL mapping studies using less dense linkage maps have confirmed their effectiveness, increasing the number of markers allows full exploitation of the recombination events in the population, improving the resolution of the QTLs. In a RIL population, where multiple meiosis could derive in short bins, this effect could notably increase not only the resolution but also the power of detection, especially for minor QTLs [15]. 
The power of detection is not comparable among different populations, but the resolution in our QTL mapping had a median QTL confidence interval of $9.42 \mathrm{cM}$ and $0.94 \mathrm{Mb}$ in genetic and physical distances, respectively. These results are comparable with the $4.04 \mathrm{cM}$ and $0.93 \mathrm{Mb}$ obtained in [15] and more precise than in other recent studies using less dense maps, where the QTL genetic confidence interval ranged between $23 \mathrm{cM}$ [5] and $28.6 \mathrm{cM}$ [4].

To validate our QTL mapping results, we used as a proof of concept two fruit quality traits that segregate in our population whose subjacent genes are already known, $\mathrm{CmOr}$ [47] and CmKFB [46] (Additional file 5: Table S2). CmOr determines the orange flesh in ripe melon when the dominant allele is present, by inducing the accumulation of $\beta$-carotene. We mapped a major QTL for CAR, CARQU9.1, at position 21,685,526 in chromosome IX, in a confidence interval of $366.8 \mathrm{~Kb}$ containing 47 genes according to the annotation version $\mathrm{v} 4.0$ of the melon genome [56] (Table 5). To observe the expression pattern of the candidate genes in this interval we used the atlas expression database Melonet-DB [57], developed using 30 different tissues from the cantalupensis variety "Harukei-3". We could reduce the number of candidate genes to 19 that were expressed in fruit flesh from 20 to $50 \mathrm{DAP}$, and only three had sequence differences between Ved and PS causing a non-synonymous amino acid change. $\mathrm{CmOr}$ was included in this final group, and the maximum LOD position of the QTL was within this gene (MELO3C005449, coordinates 21,683,406-21,690,712). CmKFB controls the biosynthesis of flavonoids in ripe melon rind, conferring the yellow external color typical of "CanaryC yellow" melons. We detected a major QTL for this trait in our RIL population, YELLQU10.1, at position 3,356,770 in chromosome X. The confidence interval of $992 \mathrm{~Kb}$ contained 156 genes (Table 5), of which 33 presented variations in our population causing a non-synonymous amino acid change. CmKFB was among them and the maximum LOD position was located approximately 100 $\mathrm{kb}$ upstream of this gene (MELO3C011980, coordinates $3,475,283-3,476,416)$.

\section{Deciphering the genetic architecture of fruit quality and domestication traits in melon}

Deciphering the genetic control of important traits in crops is one of the main objectives of modern research in agriculture. The knowledge of the responsible genes would offer the opportunity to explore the functional mechanisms that control phenotypes, allowing the search and study of allelic diversity of cultivars and accessions and ultimately to modify crop behavior. As a first step, our work identified major loci and QTLs involved in important traits in melon.

\section{Rind traits}

External color of fruit is an important trait concerning fruit quality, since the appearance is one of the main determinants for consumer choice in the market. The phenotype varies depending on the developmental stage and is determined mainly by the accumulation of pigments such as chlorophylls, carotenoids and flavonoids [58]. In our RIL population, at least two major traits control rind color: ECOL, conferring white or green rind in immature fruit, and YELL, determining the yellowing of mature rind, probably involving biosynthesis of flavonoids. Since pigment analyses was not performed, we cannot discount that other factors, such as the exposure of $\beta$-carotene after the degradation of chlorophyll due to climacteric ripening, affect the trait.

The external color of immature fruit was previously described by [44] as a monogenic trait named $W i$, but to our knowledge it has not been mapped. More recently, four loci involved in ECOL have been identified in LGs III, VII, IX and X using two mapping populations derived from PS and PI 161375, suggesting an epistatic interaction between at least some of them [59]. Our RIL population shares one parental (PS) with this study, and we mapped a minor QTL (ECOLQU3.1) and a major QTL (Wi) in the same chromosomes as ECOLQC3.5 and ECOLQC7.2 [59], respectively, however their physical positions do not co-locate (Additional file 5: Table S2). This trait has been characterized in cucumber, identifying a candidate gene, a two-component Response Regulator-like Protein (APRR2) [60, 61]. We found no $R R P$ gene in the confidence interval containing $W i$, however there was an inconsistency in the genome assembly in this region that could affect this result (Table 5). ECOLQU3.1, having a minor effect in comparison to $\mathrm{Wi}$, could slightly modify the external color by affecting the same pathway or by another mechanism.

The yellowing of mature rind has been described before, with the flavonoid naringenin chalcone identified as the principal pigment responsible for the yellow color in melon cultivars such as "Noy Amid" [58]. Recently, a Kelch domain-containing F-Box protein coding gene $(C m K F B)$ was cloned by [46], showing that this protein is the main regulator of the flavonoid biosynthetic pathway. In addition to naringenin chalcone, other downstream flavonoids have been identified in yellow melon rind. In other species, the complex of MYB-bHLH-WDR transcription factors has been shown to control flavonoid production [62, 63]. The major QTL YELLQU10.1 interval contains the gene $C m K F B$, as explained above. According to the observed 3:1 segregation for this trait (Table 1), YELLQU5.1 could act epistatically with CmKFB, regulating the biosynthesis or accumulation of naringenin chalcone or other flavonoids. Following this hypothesis, the yellow rind phenotype in our RIL population could be 
Table 5 Genomic intervals containing the identified QTLs for each trait and number of annotated genes in each interval

\begin{tabular}{|c|c|c|c|c|}
\hline Trait & Gene/QTL ID & QTL interval (cM) & QTL interval (pb) & Number of annotated genes ${ }^{a}$ \\
\hline \multirow[t]{6}{*}{ SSC } & SSCQU8.1 & 5.64 & $7,840,956$ & 389 \\
\hline & SSCQU8.2 & 2.23 & $3,935,559$ & 195 \\
\hline & SSCQU8.3 & 8.55 & $2,469,490$ & 123 \\
\hline & SSCQU9.1 & 12.68 & $3,735,902$ & 290 \\
\hline & SSCQU9.2 & 7.36 & $8,325,555$ & 525 \\
\hline & SSCQU10.1 & 19.75 & $1,445,582$ & 231 \\
\hline \multirow[t]{2}{*}{ FW } & FWQU5.1 & 6.28 & 495,756 & 48 \\
\hline & FWQU8.1 & 12.76 & $1,604,232$ & 220 \\
\hline \multirow[t]{2}{*}{ FD } & FDQU2.1 & 19.51 & $7,397,024$ & 501 \\
\hline & FDQU5.1 & 6.28 & 495,756 & 48 \\
\hline \multirow[t]{5}{*}{ FS } & FSQU2.1 & 22.77 & $1,257,435$ & 136 \\
\hline & FSQU2.2 & 6.55 & 310,997 & 34 \\
\hline & FSQU6.1 & 6.98 & $11,175,401$ & 545 \\
\hline & FSQU6.2 & 9.34 & $4,784,510$ & 432 \\
\hline & FSQU11.1 & 16.77 & $1,770,390$ & 221 \\
\hline \multirow[t]{4}{*}{$\mathrm{FL}$} & FLQU5.1 & 10.10 & 731,750 & 82 \\
\hline & FLQU6.1 & 8.11 & $10,576,960$ & 505 \\
\hline & FLQU11.1 & 19.96 & 892,764 & 108 \\
\hline & FLQU11.2 & 16.99 & $1,794,042$ & 224 \\
\hline \multirow[t]{4}{*}{ FP } & FPQU5.1 & 6.28 & 495,756 & 48 \\
\hline & FPQU6.1 & 29.43 & $25,000,330^{\mathrm{a}}$ & 1444 \\
\hline & FPQU7.1 & 16.80 & 999,749 & 149 \\
\hline & FPQU11.1 & 19.96 & 892,764 & 108 \\
\hline \multirow[t]{3}{*}{ YELL } & YELLQU5.1 & 1.11 & 295,191 & 54 \\
\hline & YELLQU10.1 (CmKFB) & 15.61 & 992,569 & 156 \\
\hline & YELLQU12.1 & 7.00 & 599,018 & 65 \\
\hline MOT & $M t-2$ & - & 858,294 & 139 \\
\hline \multirow[t]{2}{*}{ ECOL } & ECOLQU3.1 & 7.37 & $1,476,065$ & 238 \\
\hline & Wi & - & $308,385+271,774^{b}$ & $41+19$ \\
\hline CAR & CARQU9.1 (CmOr) & 2.77 & 366,884 & 47 \\
\hline SN & SNQU5.1 & 12.92 & 629,802 & 76 \\
\hline \multirow[t]{4}{*}{ SW } & SWQU3.1 & 2.99 & 423,645 & 73 \\
\hline & SWQU5.1 & 12.96 & 663,117 & 85 \\
\hline & SWQU7.1 & 4.48 & 513,375 & 90 \\
\hline & SWQU8.1 & 11.92 & 635,283 & 94 \\
\hline Median & & 9.42 & 942,667 & 130 \\
\hline
\end{tabular}

${ }^{a}$ Annotation version v4.0 of the melon genome (http://www.melonomics.net)

${ }^{\mathrm{b}} \mathrm{An}$ inconsistency between the physical and the genetic map exists in this region

determined by PS alleles in any of the two genes. Within the YELLQU5.1 interval, which contains 54 annotated genes, we identified MELO3C004621, which is described as a "Ectonucleotide pyrophosphatase/phosphodiesterase" and could be involved in the flavonoid pathway based on homology; additionally, this gene is highly expressed in fruit rind during the last stages of development in
Melonet-DB [57] and carries a variant that produced an amino acid change in the protein. However, further experiments are necessary to demonstrate the identity of YELLQU5.1.

The presence or absence of a mottled pattern also influences the rind appearance; this trait $(M t-2)$ is controlled by a major locus in LG II previously described by 
[45, 64], probably the same one that was mapped in our population (Fig. 4b). Although this pattern can be observed in other cucurbits, both the genetic control and the physiological mechanism remain unknown. One hypothesis is that the spots correspond to areas of the rind where the chlorophyll content is higher, due to an increased number, size and/or activity of chloroplasts. This hypothesis is supported by the observation of a more intense yellow in spots of mature rinds, when the allele for yellowing is present and the chlorophyll is degraded due to climacteric ripening. $M t-2$ is located in an interval of $858 \mathrm{~kb}$ that contains 139 annotated genes, without any candidate gene by functional annotation.

The rind color of fruits in our RIL population should be determined by these three traits and modified by other important aspects of fruit development, such as the type of fruit ripening (climacteric or non-climacteric), where chlorophyll degradation can be involved [65]. As discussed above, we cannot rule out that yellowing of the rind is a consequence of climacteric ripening in some of the fruits from our RIL population.

\section{Soluble solid content}

Melon fruit is mainly consumed as a dessert, a high content of sugars being a desired characteristic with special importance in crop improvement. Ved and PS are both commercial varieties on the European market, with medium-high soluble solid content, so we did not expect to find major QTLs for this trait (Table 2).

Three QTLs were found in LG VIII with LOD $>9$, between the physical positions 9,446,475 and 31,888,799 bp, explaining around $40 \%$ of the variance and the Ved allele decreasing $1.3^{\circ}$ Brix. We detected three clear peaks even increasing the confidence interval of these QTLs (Additional file 6: Figure $\mathrm{S4}$ ), but it could still be possible that there is a single QTL in this region and the low-LOD regions inside the interval were artefactual. The higher LOD value corresponds to SSCQU8.3, delimited in a region of $2.5 \mathrm{Mb}$ that contains 123 genes (Table 5). In other studies, QTLs for SSC have also been detected in LG VIII (Additional file 5: Table S2); [66] found two introgression lines (ILs) in the PS background containing introgressions from the exotic accession PI 161375 that covered the major part of chromosome VIII, including SSCQU8.3, with a significantly different SSC content.

Another three QTLs were detected in LG IX and LG $\mathrm{X}$, SCCQU9.1, SSCQU9.2 and SSCQU10.1. Although having a lower effect in SSC, they are interesting because in all cases the Ved allele increases sugar content. However, they are unstable, showing significant LOD scores only in T2, T4 and T5. QTLs for SSC in LGs IX and X have been previously described in similar positions to SSCQU9.2 and SSCQU10.1 (Additional file 5: Table S2).

\section{Fruit morphology}

Fruit morphology, including weight, size, length, diameter and shape, are key traits in the domestication process, enabling discrimination between cultivated and wild accessions. Due to their importance, they have been extensively studied in many species, especially in tomato, where several genes have been cloned (reviewed in [26]). Known genes controlling fruit size in tomato are Cell Number Regulator/FW2.2, SlKLUH/FW3.2, a cytochrome P450 A78 class, and Cell Size Regulator/FW11.3 [67]. Fruit shape is mainly determined by the combination of different alleles of $F A S$, from the YABBY family; $S U N$, an IQ domain member; $L C$, the homologue of WUS, and OVATE. In melon, with different populations used for QTL mapping studies, meta-QTLs implicated in fruit morphology have been identified $[4,59,68]$, unfortunately, none of the genes responsible for these QTLs have been cloned. All the QTLs described in the present work are supported by previous research that identified QTLs in the same LGs (Additional file 5: Table S2), except FPQU7.1. Although the physical positions associated to the QTLs are not always similar, it should be noted that only one marker was used to calculate the position of several QTLs described in Table S2 (Additional file 5), which usually span the major part of the LG. For example, $S C 5-2$ was described by [7] using an introgression line that covers almost all LG V (0-20,855,850 bp).

A clear transgressive segregation was obtained for fruit weight, from a mean of $345 \mathrm{~g}$ in RIL 172 to 1763 in RIL 140 (Additional file 7: Figure S5). Consistently, a QTL explaining $28.3 \%$ of the variation, FWQU5.1, increased the weight when the Ved allele was present. A QTL in the same chromosome has been previously described using populations developed from a cross between PS and the exotic Korean accession PI 161375 (Additional file 5: Table S2). The 496-kb interval of FWQU5.1 contains 48 genes, and among them only 23 were expressed following the expected pattern for fruit size regulators (ovary and young fruit) using the atlas expression database Melonet-DB [57]. Five of these genes carried variants in the sequence provoking a non-synonymous change between Ved and PS. One of these genes is MELO3C014402, described as FANTASTIC FOUR 2 in the annotation v4.0. These proteins are usually related to meristem development [69] and Cell Size Regulator, the gene underlying a recently cloned fruit weight QTL in tomato contains a FANTASTIC FOUR domain [67].

FLQU6.1 and FSQU6.1 are in the same region in the centromere of chromosome VI, implying that the decrease in length caused by the Ved allele provokes a decrease in the shape index. They co-localize with a QTL published recently for the same traits [68], mapped in an $\mathrm{F}_{2}$ population between PS and the Indian wild accession "Trigonus" and validated using introgression lines. In 
this case, the PS allele also increases fruit length and the percentage of variance explained is similar, around $20 \%$. The segregation of this QTL in commercial varieties suggests that it is a diversification not a domestication QTL, according to classical definitions.

Possibly, orthologs of the genes that regulate fruit size and shape in tomato could be implicated in the same process in melon, and thus be candidate genes underlying the detected QTLs. In order to evaluate whether they co-localize within the QTL intervals, we identified the potential fruit morphology orthologs in the melon genome (version 3.6.1 and annotation v4.0) (Additional file 8: Table S3A), which resulted in the identification of 89 genes. Twelve of them are contained in the intervals of FSQU2.1, FDQU2.1, FSQU6.1, FSQU6.2, FWQU8.1 and FSQU11.1 (Additional file 8: Table S3B). Among them, there are four genes (MELO3C015418, MELO3C025343, MELO3C013751, MELO3C022253) that showed the expected pattern of expression, being specific for ovaries and young fruit, according to the melon expression atlas [57]. In addition, MELO3C015418 and MELO3C025343 carried one and two non-synonymous polymorphisms between the parental lines, so they could be the candidate genes for FSQU2.1 and FDQU2.1, respectively. Additional studies, such as QTL fine mapping and differential expression analysis between Ved and PS, is needed to confirm their involvement in fruit morphology.

\section{Seed traits}

Seeds are the most valuable part of the fruit in terms of evolution, so both seed weight and number are important fitness traits. Although these traits have been widely studied in crops in which grain is consumed, such as rice [70-74] and soybean $[75,76]$, in vegetable crops much less is known. Although seed is not consumed in many vegetable crops, it is the product that seed companies commercialize. A higher seed production and better seed quality are interesting traits for both breeders and farmers.

In melon, seed size is considered a domestication trait, since wild accessions have smaller seeds than cultivated melons [3]. The genetic basis of this trait has not been well studied; a recent QTL mapping study, focused on domestication traits, evaluated seed weight, without the identification of any QTL [68], suggesting that a complex inheritance involving several minor QTLs could be the reason.

Surprisingly, in our RIL population, which is founded by two phylogenetically close commercial cultivars, we identified four QTLs for seed weight. SW was positively correlated with FP, FW and FD, but SW QTLs did not co-localize with fruit morphology QTLs (Figs. 2 and 3). Due to the high stability of the trait (Additional file 1: Figure S1), we could map these QTLs in narrow regions of the genome, spanning between 423 and $663 \mathrm{~kb}$, containing, in all cases, less than 100 genes. The stability of seed size has been studied previously in multiple crops, showing that this trait has low dispersion even in different environmental conditions, unlike seed number, which is a very plastic trait [77].

\section{Conclusions}

QTL mapping using the RIL population Ved $x$ PS identified several QTLs and major loci that modify and modulate fruit quality, from the external appearance to the biochemical composition. The location of these QTLs in narrow genomic intervals could facilitate cloning of the underlying genes and their use in breeding programs by marker-assisted selection. The introgression of favorable alleles into breeding lines could be performed easily, since the mapping population was developed from commercial cultivars, avoiding the negative consequences associated to linkage drag when using exotic material as donors. Thanks to the reduced number of annotated genes in some of these intervals, potential candidate genes have been proposed through an integrative strategy that included the analysis of gene expression and the predicted effect of variants using genomics databases.

\section{Materials and methods Plant material}

A RIL population was generated from two commercial varieties, "Védrantais" (Ved) (ssp. melo, cantalupensis group) and "Piel de Sapo" T111 (PS) (ssp. melo, inodorus group). Ved is a French variety from the group cantalupensis that produces medium-size, rounded fruits, with white external coloration when immature and cream after ripening, and with orange flesh. PS is a Spanish variety from the group inodorus, with large, elongated and mottled fruits, with dark green rind and white flesh. Both varieties have high soluble solid content since they are accepted in European markets.

The RIL population was developed in greenhouses at Cabrera de Mar (Barcelona) and Caldes de Montbui (Barcelona), through a strategy of single seed descent to $\mathrm{F}_{7}-\mathrm{F}_{8}$, starting from an $\mathrm{F}_{2}$ population obtained in 2008 . The population was composed of 89 RILs, including 82 RILs in the $F_{8}$ generation and seven in the $F_{7}$. A set of 48 polymorphic SNPs evenly distributed through the melon genome was used to confirm the homozygosity of the RILs, which was higher than $98 \%$ (not shown).

The RIL population was grown in Caldes de Montbui (Barcelona) under greenhouse conditions during the summers of 2015 (three blocks T1-T3) and 2016 (two blocks T4-T5). Plants were pruned weekly and each plant was allowed to set only a single fruit. Each block (T1-T5) contained a single individual per RIL and 1-3 individuals of the parental lines (Ved, PS) and the $\mathrm{F}_{1}$ $(\mathrm{Hyb})$. Flowers were hand-pollinated and the date was 
recorded to register the total days of development of the fruit until harvest. According to the type of ripening of each RIL, the harvest date was recorded as follows: (1) the abscission date for climacteric fruits showing abscission, (2) seven days after the first symptom of climacteric ripening (aroma production, chlorophyll degradation or abscission layer formation) for climacteric fruits that did not show abscission and (3) 60 days after pollination for non-climacteric fruits.

Blocks T1-T3 (2015) were grown during the same season but each block was separated by three weeks. Blocks T4-T5 (2016) were grown together. A mean of 8.6 lines per block were not evaluated due to problems related to seed germination, plant disease or fruit set; 60 out of 89 RILs (67\%) were evaluated in the five blocks.

\section{Phenotyping of fruit and seed traits}

Fruit quality traits (Table 6) were recorded during the development of the fruit and at harvest. Mottled rind (MOT) and external color of immature fruit (ECOL) were phenotyped as qualitative traits, before onset of ripening, around 20-30 days after pollination (DAP). Data for these traits were merged by year, due to the low variability observed among blocks (>95\% of the RILs showed the same phenotype across blocks).

After harvest, fruits were weighed and cut in two longitudinal sections: the first was scanned for morphological analysis using the Tomato Analyzer 3.0 software $[29,30]$ and the second section was used to measure soluble solid content (SSC) and total carotenoids (CAR). The morphology traits recorded were fruit weight (FW), diameter (FD), length (FL) and perimeter (FP) with the Tomato Analyzer 3.0 software, and shape index (FS) was calculated as the ratio between FL and FD. To measure SSC, four flesh samples of $1 \mathrm{~cm}$ diameter per melon were used; the juice was extracted by pressuring the

Table 6 Traits evaluated in the "Védrantais" x "Piel de Sapo" Recombinant Inbred Line population

\begin{tabular}{ll}
\hline Trait & Code \\
\hline Soluble solid content & SSC \\
Fruit weight & FW \\
Fruit diameter & FD \\
Fruit shape & FS \\
Fruit length & FL \\
Fruit perimeter & FP \\
Yellowing of mature rind & YELL \\
Mottled rind & MOT \\
External color of immature fruit & ECOL \\
Total carotenoid content & CAR \\
Seed weight & SW \\
Seed number & SN \\
\hline
\end{tabular}

samples against a strainer and analyzed with a digital hand refractometer (Atago Co. Ltd., Tokyo, Japan). Total carotenoid analysis was performed by UV-VIS Spectroscopy [31] as described by [32], using the flesh from the first three blocks (T1-T3). The yellowing of mature rind (YELL) was recorded as a qualitative trait (0 absence, 1 presence) by visual inspection of ripe fruits. Fifteen dried seeds were used to estimate seed weight (SW) and seed number (SN) per fruit.

\section{Genotyping and linkage map construction}

Young leaves from the RIL population and the parental lines Ved and PS were collected during the summer of 2015 and stored at $-80^{\circ} \mathrm{C}$. DNA extraction was following the CTAB protocol [33] with some modifications. Briefly, the isopropanol precipitation was followed by incubating $30 \mathrm{~min}$ at $4{ }^{\circ} \mathrm{C}$ and centrifuging for $10 \mathrm{~min}$ after adding the washing buffer. The extracted DNA was re-suspended in MilliQ water. PicoGreen ${ }^{\circ}$ dsDNA Assay Kit (Life Technologies) was used according to the manufacturer's protocol for quantity assessment, and DNA quality was estimated by gel electrophoresis.

GBS was performed at the National Center of Genomic Analyses (CNAG, Barcelona, Spain). ApeKI GBS libraries of the 89 RIL and the parental lines (PS and Ved) were prepared at CNAG and sequenced using Illumina HiSeq2000 $(2 \times 100$ bp).

The Fast-GBS pipeline [34] and the melon v3.6.1 genome assembly (http://www.melonomics.net) were used to identify the variants (SNPs and INDELs). Fast-GBS uses the maximal exact matches algorithm implemented in BWA for alignment of the reads, and relies on the Platypus software [35] for variant calling. The parameters applied were the following: minimum number of reads per locus (default $=2$ ); mapping quality score of reads to call a variant $(\mathrm{MQ} \geq 20)$; minimum base quality (20); multiple nucleotide polymorphisms distance (minFlank =5), and maximum missing data allowed (default $\leq 80 \%$ ). As a preliminary check, the row data were cross-checked with a set of variants identified after the whole genome re-sequencing of the two parental lines PS and Ved [36], with the overlapping variants retained (pre-filtered variants) and used in the downstream analyses. Vcftools [37] and in-house scripts were subsequently applied to retain only bi-allelic variants with a Minor Allele Count greater than 1, with at least one homozygous variant for marker, with a global quality greater than 100 and with missing values $(\mathrm{MV}) \leq 60 \%$. In addition, sparse heterozygous variants with a genotype depth lower than 5 were converted to missing values.

Once variants were obtained and filtered, a chromosome-by-chromosome manual inspection was performed and variants showing highly distorted segregation and discrepancies with the genotyping of the 
parental lines were discarded. This final set of variants was used to define the bins. A bin was established as a group of variants with the same genotype for each RIL, meaning a region without any recombination breakpoint in any individual of the population. A single variant with the lowest number of missing data was selected to represent each bin. If missing data were present in this variant, they were imputed manually, recovering the genotyping information from the other variants in the same bin. Finally, only bins with less than $30 \%$ of missing data were considered valid to generate the genetic map. Bins with a discrepancy between genetic and physical maps but showing a proper segregation and reliable quality were included in the genetic map construction.

The genetic linkage map was constructed using the online software tool MSTmap [38]. Linkage groups (LG) were formed at minimum $L O D=10$ and we allowed the software to detect genotyping errors. Mapping size threshold was set to 2 and the distance threshold to 15 $\mathrm{cM}$. The genetic distances were estimated using the Kosambi mapping function [39]. The graphical representation of the genetic map was obtained using MapChart version $2.2[40]$.

\section{QTL mapping}

For quantitative traits, we were aware that environmental effects can have a considerable influence on the phenotype. A Principal Component Analysis (PCA) was used to test if the quantitative data of the blocks from the same year could be merged, creating only one dataset per year (Fig. 1a). We could not identify a pattern that distinguished the blocks according to the year, in fact $\mathrm{T} 1$ and $\mathrm{T} 5$ were very similar even though they were from different years. Considering these results, we decided to analyze the data in two different ways: one including the mean for each line after merging data from the five blocks, and the other including the individual data for each block.

The QTL mapping was performed with MapQTL6 [41] considering each block (T1-T5) and the mean across the blocks. We used the interval mapping procedure for all traits, and the Kruskal-Wallis (KW) test for the monogenic and oligogenic traits. QTLs in the mapping experiment with the mean values for LOD $>2.5$ were considered significant. To evaluate their significance in the individual blocks we checked if they were located in the same position and with the same positive or negative additive effect.

The identified QTL, were coded with the terminology system described in [21], where the first letters represent the trait abbreviation, followed by a "Q" for QTL, a letter for the mapping experiment (" $U$ ", in this case), a digit designating the linkage group (LG) to which the QTL maps, a dot and a final number to differentiate QTL present in the same LG.

\section{Statistical analyses}

The statistical analyses and graphical representations were performed using the software R v3.2.3 [42] with the RStudio v1.0.143 interface [43].

The normality of distributions was tested using the Shapiro-Wilk test, assuming that it was significantly different from a normal distribution when $p$-value $<0.05$.

The R package "factoextra" was used for PCA, with data from a random subset of 22 RILs which had no missing values in any block and a representative subset of seven quantitative variables (SSC, FW, FL, FD, FS, SN and SW). We removed the line effect to observe only the effect of the block (environment) before the PCA, using the "removeBatchEffect" function from the R package "limma".

To obtain the correlation matrix among traits we calculated the Pearson coefficient with the $\mathrm{R}$ package "Hmisc" with "corrplot".

\section{Additional files}

Additional file 1: Figure S1. Distribution of the quantitative traits evaluated in the parental lines PS, Ved and Hyb. Each dot corresponds to an observation in any of the five blocks T1-T5. The mean for each line is shown with a horizontal line. (TIF $783 \mathrm{~kb}$ )

Additional file 2: Figure S2. Distribution of the quantitative traits evaluated in the RIL population for each block (T1-T5). Black stars indicate a significant deviation from normality. (TIF $2161 \mathrm{~kb}$ )

Additional file 3: Table S1. Markers obtained with GBS. (A) All markers; (B) Selected markers; (C) All bins; (D) Selected bins. (XLSX $5125 \mathrm{~kb}$ )

Additional file 4: Figure S3. Examples of complicated cases to phenotype. a. Mottled rind (MOT) is partially masked in PS due to the dark green rind, but darker spots can be observed in comparison to RIL 196A. b. Yellowing of mature rind (YELL), present in RIL 190 and appreciable as a different tone in green rind. (TIF 9290 kb)

Additional file 5: Table S2. Summary of QTLs described in other studies that map in similar intervals to those detected here. (PDF $80 \mathrm{~kb}$ )

Additional file 6: Figure S4. LOD scores for SSC in the QTL mapping experiment using the mean values for a QTL region in LG VIII. The dots represent the LOD peak for each QTL (SSCQU8.1, SSCQU8.2 and SSCQU8.3 from left to right) and the bars the LOD peak \pm 1.5 . (TIF $159 \mathrm{~kb}$ )

Additional file 7: Figure S5. RILs 172 (a) and 140 (b) from 2016, showing transgressive segregation in fruit size in comparison with the parental lines PS (c) and Ved (d). The white square represents $1 \mathrm{~cm}^{2}$. (TIF $9704 \mathrm{~kb}$ )

Additional file 8: Table S3. (a) Candidate genes for fruit morphology in the melon genome annotation v4.0. (b). Candidate genes for fruit morphology contained in the QTL intervals. (XLSX 19 kb)

Additional file 9: Phenotyping data values for each trait. (TXT $5 \mathrm{~kb}$ )

Additional file 10: Genotyping data. (TXT $157 \mathrm{~kb}$ )

Additional file 11: Linkage group positions for each marker. (TXT $18 \mathrm{~kb}$ )

\section{Abbreviations}

CAR: Total carotenoids; DAP: Days after pollination; ECOL: External color of immature fruit; FD: Fruit diameter; FL: Fruit length; FP: Fruit perimeter; FS: Shape index; FW: Fruit weight; GBS: Genotyping-by-sequencing; IL: Introgression line; KW: Kluskal-Wallis; LG: Linkage group; MOT: Mottled rind; MQ: Mapping quality; MV: Missing value; NGS: Next-generation sequencing; PCA: Principal component analysis; PS: Piel de sapo;

QTL: Quantitative trait locus; RIL: Recombinant inbred line; SN: Seed number; SSC: Soluble solid content; SW: Seed weight; VED: Védrantais; YELL: Yellowing of mature rind 


\section{Acknowledgements}

We thank Pere Arús for helpful suggestions in the genetic map construction.

\section{Funding}

This work was supported by the Spanish Ministry of Economy and Competitiveness grant AGL2015-64625-C2-1-R, Severo Ochoa Programme for Centres of Excellence in R\&D 2016-2010 (SEV-2015-0533) and the CERCA Programme/Generalitat de Catalunya to JGM. LP was supported by a FPI grant from the Spanish Ministry of Economy and Competitiveness. VR was supported by the European Union's Horizon 2020 research and innovation programme under Marie Skłodowska-Curie grant agreement No 6655919.

\section{Availability of data and materials}

All the data of the present study have been included in tables and/or figures. Mapping data are also provided in Additional files 9, 10 and 11 .

\section{Authors' contributions}

LP, MF and TJ obtained the RIL population. LP cultivated and phenotyped the RIL population with the contribution of SP. VR and KGA analyzed the GBS data. LP constructed the genetic map and performed the QTL analyses. MP and JGM conceived, designed and coordinated the study. LP, VR, MP and JGM drafted the manuscript. All authors read and approved the final manuscript.

\section{Ethics approval and consent to participate}

Not applicable.

\section{Consent for publication}

Not applicable.

\section{Competing interests}

The authors declare that they have no competing interests.

\section{Publisher's Note}

Springer Nature remains neutral with regard to jurisdictional claims in published maps and institutional affiliations.

\section{Author details}

${ }^{1}$ Centre for Research in Agricultural Genomics (CRAG) CSIC-IRTA-UAB-UB, Campus UAB, 08193 Cerdanyola, Barcelona, Spain. ${ }^{2}$ IRTA (Institut de Recerca i Tecnologia Agroalimentàries), Campus UAB, 08193 Cerdanyola, Barcelona, Spain. ${ }^{3}$ Semillas Fitó S.A., 08348 Cabrera de Mar, Barcelona, Spain.

Received: 6 March 2018 Accepted: 19 November 2018 Published online: 04 December 2018

\section{References}

1. FAO. Statistics Division of Food and Agriculture Organization of the United Nations (FAOSTAT). URL http://faostat.fao.org/.

2. Sebastian P, Schaefer H, Telford IRH, Renner SS. Cucumber (Cucumis sativus) and melon (C. melo) have numerous wild relatives in Asia and Australia, and the sister species of melon is from Australia. Proc Natl Acad Sci USA. 2010; 107:14269-73.

3. Pitrat M. Phenotypic diversity in wild and cultivated melons (Cucumis melo). Plant Biotechnol. 2013:30:273-8.

4. Díaz A, Zarouri B, Fergany M, Eduardo I, Álvarez JM, Picó B, et al. Mapping and Introgression of QTL Involved in Fruit Shape Transgressive Segregation into "Piel de Sapo" Melon (Cucumis melo L.). PLoS One. 2014;9:e104188.

5. Ramamurthy RK, Waters BM. Identification of fruit quality and morphology QTLs in melon (Cucumis melo) using a population derived from flexuosus and cantalupensis botanical groups. Euphytica. 2015;204:163-77.

6. Perpiñá G, Esteras C, Gibon Y, Monforte AJ, Picó B. A new genomic library of melon introgression lines in a cantaloupe genetic background for dissecting desirable agronomical traits. BMC Plant Biol. 2016;16:1-21.

7. Eduardo I, Arús P, Monforte AJ. Development of a genomic library of near isogenic lines (NILs) in melon (Cucumis melo L.) from the exotic accession PI161375. Theor. Appl. Genet. 2005;112:139-48.

8. Périn C, Gomez-Jimenez M, Hagen L, Dogimont C, Pech J, Latché A, et al. Molecular and genetic characterization of a non- climacteric phenotype in melon reveals two loci conferring altered ethylene response in fruit. Plant Physiol. 2002;129:300-9.
9. Freilich S, Lev S, Gonda I, Reuveni E, Portnoy V, Oren E, et al. Systems approach for exploring the intricate associations between sweetness, color and aroma in melon fruits. BMC Plant Biol. 2015;15:71.

10. Galpaz N, Gonda I, Shem-Tov D, Barad O, Tzuri G, Lev S, et al. Deciphering genetic factors that determine melon fruit-quality traits using RNA-Seq based high-resolution QTL and eQTL mapping. Plant J. 2018;94:169-191. https://doi.org/10.1111/tpj.13838

11. Gur A, Tzuri G, Meir A, Sa U, Portnoy V, Katzir N, et al. Genome-wide linkagedisequilibrium mapping to the candidate gene level in melon (Cucumis melo). Sci Rep. 2017;7:1-13.

12. Leida C, Moser C, Esteras C, Sulpice R, Lunn JE, de Langen F, et al. Variability of candidate genes, genetic structure and association with sugar accumulation and climacteric behavior in a broad germplasm collection of melon (Cucumis melo L.). BMC Genet. 2015;16:28.

13. Garcia-Mas J, Benjak A, Sanseverino W, Bourgeois M, Mir G, González VM, et al. The genome of melon (Cucumis melo L.). Proc Natl Acad Sci U.S.A. 2012; 109:11872-7.

14. Nimmakayala P, Tomason YR, Abburi VL, Alvarado A, Ronin Y, Garcia-Mas J, et al. Genome-wide differentiation of various melon horticultural groups for use in GWAS for fruit firmness and construction of a high resolution genetic map. Front Plant Sci. 2016:7:1-15.

15. Chang C, Wang Y, Tung C. Genome-wide single nucleotide polymorphism discovery and the construction of a high-density genetic map for melon (Cucumis melo L.) using genotyping-by-sequencing. Front. Plant Sci. 2017;8:1-11.

16. Elshire RJ, Glaubitz JC, Sun Q, Poland JA, Kawamoto K, Buckler ES, et al. A robust, simple genotyping-by-sequencing (GBS) approach for high diversity species. PLoS One. 2011;6:e19379.

17. Huang Y, Poland JA, Wight CP, Jackson EW, Tinker NA. Using genotypingby-sequencing (GBS) for genomic discovery in cultivated oat. PLoS One. 2014:9:e102448.

18. Liu H, Bayer M, Druka A, Russell JR, Hackett CA, Poland J, et al. An evaluation of genotyping by sequencing (GBS) to map the Breviaristatum-e (ari-e) locus in cultivated barley. BMC Genomics. 2014;15:1-11.

19. Celik I, Gurbuz N, Uncu AT, Frary A, Doganlar S. Genome-wide SNP discovery and QTL mapping for fruit quality traits in inbred backcross lines (IBLs) of Solanum pimpinellifolium using genotyping by sequencing. BMC Genomics. 2017;18:1

20. Wei Q, Wang Y, Qin X, Zhang Y, Zhang Z, Wang J, et al. An SNP-based saturated genetic map and QTL analysis of fruit-related traits in cucumber using specific length amplified fragment (SLAF) sequencing. BMC Genomics. 2014;15:1-10.

21. Diaz A, Fergany M, Formisano G, Ziarsolo P, Blanca J, Fei Z, et al. A consensus linkage map for molecular markers and quantitative trait loci associated with economically important traits in melon (Cucumis melo L.). BMC Plant Biol. 2011;11:111.

22. Harel-Beja R, Tzuri G, Portnoy V, Lotan-Pompan M, Lev S, Cohen S, et al. A genetic map of melon highly enriched with fruit quality QTLs and EST markers, including sugar and carotenoid metabolism genes. Theor Appl Genet. 2010;121:511-33.

23. Pitrat M. Melon genetic resources: phenotypic diversity and horticultural taxonomy. New York: Springer New York; 2016. p. 1-36.

24. Dogimont C. 2011 gene list for melon. Cucurbit Genet Coop Rep. 2011;3334:104-33.

25. Vegas J, Garcia-Mas J, Monforte AJ. Interaction between OTLs induces an advance in ethylene biosynthesis during melon fruit ripening. Theor Appl Genet. 2013;126:1531-44.

26. Monforte AJ, Diaz A, Caño-Delgado A, Van Der Knaap E. The genetic basis of fruit morphology in horticultural crops: lessons from tomato and melon. J Exp Bot. 2014;65:4625-37.

27. Argyris JM, Díaz A, Ruggieri V, Fernández M, Jahrmann T, Gibon Y, et al. QTL analyses in multiple populations employed for the fine mapping and identification of candidate genes at a locus affecting sugar accumulation in melon (Cucumis melo L). front. Plant Sci. 2017:8:1-20.

28. Zamir D. Improving plant breeding with exotic genetic libraries. Nat Rev Genet. 2001:2:3-10.

29. Brewer MT, Lang L, Fujimura K, Dujmovic N, Gray S, Van Der Knaap E. Development of a controlled vocabulary and software application to analyze fruit shape variation in tomato and other plant species. Plant Physiol. 2006;141:15-25.

30. Darrigues A, Hall J, Van Der Knaap E, Francis DM, Dujmovic N, Gray S. Tomato analyzer-color test : a new tool for efficient digital phenotyping. J Am Soc Hortic Sci. 2008;133:579-86. 
31. Lichtenthaler H, Buschmann C. Chlorophylls and carotenoids: measurement and characterization by UV-VIS spectroscopy. Curr Protoc Food Anal Chem. 2001:F4.3.1-8.

32. Perelló C, Rodríguez-Concepción M, Pulido P. Quantification of plant resistance to isoprenoid biosynthesis inhibitors. In: Rodríguez-Concepción M, editor. Plant isoprenoids. Methods in molecular biology (methods and protocols), vol 1153. New York: Humana Press. 2014.

33. Doyle J. DNA Protocols for Plants. In: Hewitt GM, AWB J, JPW Y, editors. Mol. Tech. Taxon. Berlin: Springer Berlin Heidelberg; 1991. p. 283-93.

34. Torkamaneh D, Laroche J, Bastien M, Abed A, Belzile F. Fast-GBS: a new pipeline for the efficient and highly accurate calling of SNPs from genotyping-by-sequencing data. BMC Bioinformatics. 2017;18:1-7.

35. Rimmer A, Phan H, Mathieson I, lqbal Z, Twigg SRF, Consortium WGS, et al. Integrating mapping-, assembly- and haplotype-based approaches for calling variants in clinical sequencing applications. Nat Genet. 2014; 46:912-8.

36. Sanseverino W, Hénaff E, Vives C, Pinosio S, Burgos-Paz W, Morgante M, et al. Transposon insertions, structural variations, and SNPs contribute to the evolution of the melon genome. Mol Biol Evol. 2015;32:2760-74.

37. Danecek P, Auton A, Abecasis G, Albers CA, Banks E, Depristo MA, et al. The variant call format and VCFtools. Bioinformatics. 2017;27:2156-8.

38. Wu Y, Bhat PR, Close TJ, Lonardi S. Efficient and accurate construction of genetic linkage maps from the minimum spanning tree of a graph. PLoS Genet. 2008:4(10):e1000212.

39. Kosambi DD. The estimation of map distances from recombination values. Ann Eugenics. 1944;12:172-5.

40. Voorrips RE. MapChart: software for the graphical presentation of linkage maps and QTLs. J Hered. 2002;93:77-8.

41. Van Ooijen J, Maliepaard C. MapQTL Version 3.0: Software for the calculation of QTL positions on genetic maps. DLO-Centre Plant Breed. Reprod Res Wageningen, Netherlands. 1996.

42. R Core Team. R: A language and environment for statistical computing. Vienna: R Foundation for Statistical Computing; 2012. URL http://www.Rproject.org/

43. RStudio Team. RStudio: Integrated Development for R. RStudio, Inc., Boston, MA; 2015. URL http://www.rstudio.com/

44. Kubicki B. Inheritance of some characters in muskmelons (Cucumis melo). Genet Pol. 1962;3:265-74.

45. Périn C, Dogimont C, Giovinazzo N, Besombes D, Guitton L, Hagen L, et al. Genetic control and linkages of some fruit characters in melon. Cucurbit Genet Coop Rep. 1999;22:16-8.

46. Feder A, Burger J, Gao S, Lewinsohn E, Katzir N, Schaffer AA, et al. A Kelch domain-containing F-box coding gene negatively regulates flavonoid accumulation in muskmelon. Plant Physiol. 2015:169:1714-26.

47. Tzuri G, Zhou X, Chayut N, Yuan H, Portnoy V, Meir A, et al. A "golden" SNP in CmOr governs the fruit flesh color of melon (Cucumis melo). Plant J. 2015; 82:267-79.

48. Ferreira A, Flores da Silva M, Da Costa e Silva L, Cruz CD. Estimating the effects of population size and type on the accuracy of genetic maps. Genet Mol Biol. 2006;29:187-92

49. Chang C, Bowman JL, Dejohn AW, Landert ES, Meyerowitz EM. Restriction fragment length polymorphism linkage map for Arabidopsis thaliana. Proc Natl Acad Sci U S A. 1988:85:6856-60.

50. Tanksley SD, Medina-Filho H, Rick CM. Use of naturally-occurring enzyme variation to detect and map genes controlling quantitative traits in an interspecific backcross of tomato. Heredity. 1982;49:11-25.

51. Tanksley SD, Ganal MW, Prince JP, de Vicente MC, Bonierbale MW, Broun P, et al. High density molecular linkage maps of the tomato and potato genomes. Genetics. 1992;132:1141-60.

52. June C, Page SEEL, Pasternak S, Liang C, Zhang J, Fulton L, et al. The B73 maize genome: complexity, diversity, and dynamics. Science. 2012;326 1112-5.

53. Goff SA, Ricke D, Lan T, Presting G, Wang R, Dunn M, et al. A draft sequence of the Rice genome (Oryza sativa L. ssp japonica). Science. 2005;296:92-100.

54. The Tomato Genome Consortium. The tomato genome sequence provides insights into fleshy fruit evolution. Nature. 2012:485:635-41.

55. Pavan S, Marcotrigiano AR, Ciani E, Mazzeo R, Zonno V, Ruggieri V, et al. Genotyping-by-sequencing of a melon (Cucumis melo L.) germplasm collection from a secondary center of diversity highlights patterns of genetic variation and genomic features of different gene pools. BMC Genomics. 2017;18:1-10.
56. Ruggieri V, Alexiou KG, Morata J, et al. An improved assembly and annotation of the melon (Cucumis melo L.) reference genome. Sci Rep. 2018;8:8088

57. Yano R, Nonaka S, Ezura H. Melonet-DB, A Grand RNA-seq gene expression atlas in melon (Cucumis melo L.). Plant Cell Physiol. 2018;59(1):e4.

58. Tadmor Y, Burger J, Yaakov I, Feder A, Libhaber SE, Portnoy V, et al. Genetics of flavonoid, carotenoid, and chlorophyll pigments in melon fruit rinds. J Agric Food Chem. 2010;58:10722-8.

59. Monforte AJ, Oliver M, Gonzalo MJ, Alvarez JM, Dolcet-Sanjuan R, Arús P. Identification of quantitative trait loci involved in fruit quality traits in melon (Cucumis melo L.). Theor Appl Genet. 2004;108:750-8.

60. Liu H, Meng H, Pan Y, Liang $X$, Jiao J, Li Y, et al. Fine genetic mapping of the white immature fruit color gene $w$ to a $33.0-k b$ region in cucumber (Cucumis sativus L.). Theor Appl Genet. 2015;128:2375-85.

61. Liu H, Jiao J, Liang X, Liu J, Meng H, Chen S, et al. Map-based cloning, identification and characterization of the $w$ gene controlling white immature fruit color in cucumber (Cucumis sativus L.). Theor Appl Genet. 2016;129:1247-56.

62. Xu W, Dubos C. Transcriptional control of flavonoid biosynthesis by MYB bHLH - WDR complexes. Trends Plant Sci. 2015;20:176-85.

63. Xu W, Grain D, Bobet S. Complexity and robustness of the flavonoid transcriptional regulatory network revealed by comprehensive analyses of MYB - bHLH - WDR complexes and their targets in Arabidopsis seed. New Phytol. 2014;202:132-44.

64. Ganesan J. Genetic studies on certain characters of economic importance in muskmelon (Cucumis melo L.). Dissertation Annamalai Univ. 1988.

65. Pech JC, Bouzayen M, Latché A. Climacteric fruit ripening: ethylenedependent and independent regulation of ripening pathways in melon fruit. Plant Sci. 2008;175:114-20.

66. Eduardo I, Arús P, Monforte AJ, Obando J, Fernández-Trujillo JP, Martínez JA, et al. Estimating the genetic architecture of fruit quality traits in melon using a genomic library of near isogenic lines. J Amer Soc Hort Sci. 2007; 132:80-9.

67. Mu Q, Huang Z, Chakrabarti M, Illa-Berenguer E, et al. Fruit weight is controlled by cell size regulator encoding a novel protein that is expressed in maturing tomato fruits. PLoS Genet. 2017;13:e1006930.

68. Díaz A, Hernández M, Dolcet R, Garcés A, José C, Álvarez M, et al. Quantitative trait loci analysis of melon (Cucumis melo L.) domesticationrelated traits. Theor Appl Genet. 2017;130:1837-56.

69. Wahl V, Brand LH, Guo Y, Schmid M. The FANTASTIC FOUR proteins influence shoot meristem size in Arabidopsis thaliana. BMC Plant Biol. 2010; 10:1-12.

70. Duan P, Xu J, Zeng D, Zhang B, Geng M, Zhang G, et al. Natural variation in the promoter of GSE5 contributes to grain size diversity in Rice. Mol Plant. 2017;10:685-94.

71. Fan C, Xing $\mathbb{E} Y$, Mao $\mathbb{E H}$. GS3, a major QTL for grain length and weight and minor QTL for grain width and thickness in rice, encodes a putative transmembrane protein. Theor Appl Genet. 2006;112:1164-71.

72. Shomura A, Izawa T, Ebana K, Ebitani T, Kanegae H, Konishi S, et al. Deletion in a gene associated with grain size increased yields during rice domestication. Nat Genet. 2008;40:1023-8.

73. Song $X$, Huang W, Shi M, Zhu M, Lin H. A QTL for rice grain width and weight encodes a previously unknown RING-type E3 ubiquitin ligase. Nat Genet. 2007;39:623-30.

74. Wang S, Wu K, Yuan Q, Liu X, Liu Z, Lin X, et al. Control of grain size, shape and quality by OSSPL16 in rice. Nat Genet. 2012;44:950-4.

75. Lu X, Xiong Q, Cheng T, Li Q, Liu X, Bi Y, et al. A PP2C-1 allele underlying a quantitative trait locus enhances soybean 100-seed weight. Mol Plant. 2017; 10:670-84.

76. Tang X, Su T, Han M, Wei L, Wang W, Yu Z, et al. Suppression of extracellular invertase inhibitor gene expression improves seed weight in soybean (Glycine max). J Exp Bot. 2017;68:469-82.

77. Fisher J, Bensal E, Zamir D. Bimodality of stable and plastic traits in plants. Theor Appl Genet. 2017;130:1915-26. 